\title{
LFV and (g-2) in non-universal SUSY models with light higgsinos
}

\author{
C. Han, ${ }^{a}$ M.L. López-lbáñez, ${ }^{b}$ A. Melis, ${ }^{c}$ O. Vives, ${ }^{c}$ L. Wu ${ }^{d}$ and J.M. Yang ${ }^{b, e}$ \\ ${ }^{a}$ School of Physics, KIAS, \\ 85 Hoegiro, Seoul 02455, Republic of Korea \\ ${ }^{b}$ CAS Key Laboratory of Theoretical Physics, Institute of Theoretical Physics, \\ Chinese Academy of Sciences, \\ Zhong Guan Cun East Street 55, Beijing 100190, P.R. China \\ ${ }^{c}$ Departament de Fúsica Tèorica, Universitat de València \& IFIC, Universitat de València \& CSIC, \\ Dr. Moliner 50, E-46100 Burjassot (València), Spain \\ ${ }^{d}$ Department of Physics and Institute of Theoretical Physics, Nanjing Normal University, \\ Wenyuan Road 1, Nanjing 210023, P.R. China \\ ${ }^{e}$ School of Physical Sciences, University of Chinese Academy of Sciences, \\ Yuquan Road 10A, Beijing 100049, P.R. China \\ E-mail: hancheng@itp.ac.cn, maloi2@uv.es, aurora.melis@uv.es, \\ oscar.vives@uv.es, leiwu@itp.ac.cn, jmyang@itp.ac.cn
}

ABSTRACT: We consider a supersymmetric type-I seesaw framework with non-universal scalar masses at the GUT scale to explain the long-standing discrepancy of the anomalous magnetic moment of the muon. We find that it is difficult to accommodate the muon g-2 while keeping charged-lepton flavor violating processes under control for the conventional $\mathrm{SO}(10)$-based relation between the up sector and neutrino sector. However, such tension can be relaxed by adding a Georgi-Jarlskog factor for the Yukawa matrices, which requires a non-trivial GUT-based model. In this model, we find that both observables are compatible for small mixings, CKM-like, in the neutrino Dirac Yukawa matrix.

Keywords: Precision QED, Supersymmetric Standard Model, GUT, Neutrino Physics

ARXIV EPRINT: 2003.06187 


\section{Contents}

1 Introduction 1

2 Supersymmetric type-I seesaw $\quad 3$

$\begin{array}{lll}3 & \text { RGEs and lepton flavour violation } & 6\end{array}$

4 Observables $\quad 7$

4.1 Higgs mass 7

4.2 Anomalous magnetic moment of the muon $\quad 7$

4.3 CLFV observables 9

4.4 $\operatorname{BR}(\mu \rightarrow \mathrm{e} \gamma)$ versus $a_{\mu}^{\text {susy }} \quad 10$

5 Analysis $\quad \mathbf{1 2}$

5.1 Lepton observables 13

$\begin{array}{ll}5.2 \text { Benchmark point } & 16\end{array}$

$\begin{array}{llr}6 & \text { Conclusions } & 18\end{array}$

$\begin{array}{lr}\text { A Loop functions } & 19\end{array}$

$\begin{array}{lr}\text { B Mass insertion approximation } & 19\end{array}$

$\begin{array}{ll}\text { B.1 Flavour-expansion theorem } & 20\end{array}$

B.2 Expressions for $\operatorname{BR}(\mu \rightarrow \mathrm{e} \gamma)$ and $a_{\mu}^{\text {susy }} 20$

C Majorana mass matrix 23

\section{Introduction}

After the discovery of neutrino oscillations in 1998 by Superkamiokande [1, 2], the Standard Model (SM) was forced to include massive neutrinos. Yet, the smallness of their masses seems to require a new framework different from the SM Yukawa couplings in the charged lepton or quark sectors. Simultaneously, it was confirmed experimentally that neutrino mass eigenstates are a non-trivial combination of the flavour states. Consequently, we know that the family lepton numbers, $L_{\mathrm{e}}, L_{\mu}$ and $L_{\tau}$, are violated in Nature. This necessarily implies some degree of violation in the charged-lepton sector, although it has not yet been observed.

Several mechanisms have been concocted to explain the extreme smallness of neutrino masses and all of them require the existence of new physics (NP). Unfortunately, the available experimental information on the mass splittings and mixing is still insufficient to 
disclose the physics behind their true origin. New observables are required to improve our understanding and charged-lepton-flavour violation (CLFV) is one of the best options at our reach. Nevertheless, the absence of any signal of CLFV may indicate that the associated NP is considerably heavy. On the other hand, the persistent discrepancy between the experimental measurement of the anomalous magnetic moment of the muon [3] and its SM prediction [4-8],

$$
\Delta a_{\mu}=a_{\mu}^{\exp }-a_{\mu}^{\mathrm{SM}}=(2.7 \pm 0.7) \times 10^{-9},
$$

remains an interesting motivation to explore new models [9-14], for a review see [15]. ${ }^{1}$

With respect to neutrino masses, the type-I seesaw mechanism [19-24] seems to be the most natural extension of the SM to generate them. It assumes the existence of right-handed neutrinos $(\mathrm{RH} \nu)$, which are singlets of the SM and, therefore, are allowed to have both Dirac and Majorana mass terms by the gauge symmetries. Mediated by them, an effective dimension-5 Weinberg operator [25] would be induced, producing the light neutrino masses after the electroweak (EW) symmetry breaking. Although $\mathrm{RH} \nu$ may live at any scale between the EW and the GUT scale, a naïve dimensional analysis of the Weinberg operator signals a Majorana mass around the usual GUT scale $\sim 10^{15-16} \mathrm{GeV}$. That means that the SM supplemented with $\mathrm{RH} \nu$ at high energies suffers from a serious hierarchy problem [26, 27].

Despite the fact that no signal of superparticles has been found at LHC, supersymmetric models may still be the appropriate candidate to alleviate this problem and may also answer other questions, such as the nature of dark matter $[28,29]$ and the exact unification of the gauge couplings at the GUT scale [30-33]. In addition, the presence of right-handed neutrinos induces slepton mixing through the renormalization group equation (RGE), which may produce visible CLFV effects [34-37]. In particular, SUSY models with non-universal scalar masses at the GUT scale seem to be favoured from naturalness considerations [3840], allowing for a Higgs boson mass at $125 \mathrm{GeV}$ and low electroweak fine-tuning [41, 42]. Here we will analyse supersymmetric non-universal Higgs models with an additional parameter for the third generation of scalar superpartners (NUHM3) [43-45]. We focus on the so-called light higgsino-world scenario [46-55] in which the SUSY matter scalars are pushed into the multi-TeV scale while $\mu \lesssim 1 \mathrm{TeV}$, as natural SUSY requires. We observe that a characteristic spectrum where the lightest neutralinos and chargino are higgsino-like is automatically selected when the correct $(g-2)_{\mu}$ phenomenology is required through a general scan that includes solutions with the usual gaugino-higgsino hierarchy. This light-higgsino spectrum is favoured by naturalness and also by dark matter searches, as higgsino is efficiently annihilated, unlike the typical bino-like dark matter that is usually over-abundant.

In the literature, refs. $[56,57]$ present related analysis, although following a different approach. In [56], the authors studied the preferred regions of the supersymmetric parameter space according to the muon g-2, implementing the LHC bounds from the Run-I

\footnotetext{
${ }^{1} \mathrm{~A}$ recent lattice calculation [16] renders a $(g-2)_{\mu}$ result that, if confirmed, will lead to a disappearance of the discrepancy and the role of new physics in it. Yet, other works $[17,18]$ argument that this shift in the value of the anomalous magnetic moment has important consequences in the EW fit so that other observables will inherit the need for new physics with a similar significance. We will discuss the potential impact of this result on our analysis in section 5.1.
} 
but imposing no low-energy constraints from CLFV. In contrast, the second article analysed the CLFV processes for NUHM3 models but did not comment on the possibility of reproducing $(g-2)_{\mu}$.

The paper is organised as follows: in section 2, the main ingredients of a supersymmetric type-I seesaw model are presented. We also comment on some specific features common to GUT-motivated scenarios. In section 3, the effect of the right-handed neutrinos on the running of the slepton soft masses is described. In section 4, we discuss the main observables of our analysis providing some useful analytic relations between CLFV processes and $(g-2)_{\mu}$. Section 5 is devoted to the results of our numerical scan. We summarize our conclusions in section 6 .

\section{Supersymmetric type-I seesaw}

The supersymmetric type-I seesaw considers the MSSM particle content augmented with three chiral superfields, one for each generation of right-handed neutrinos $(\mathrm{RH} \nu)$. The interactions among chiral supermultiplets are determined by the superpotential which, in this framework, contains new interactions involving $\mathrm{RH} \nu$ :

$$
W=W_{\mathrm{MSSM}}+\nu_{R}^{c T} Y_{\nu} \ell_{L} \cdot H_{u}+\frac{1}{2} \nu_{R}^{c T} M_{R} \nu_{R}^{c},
$$

with

$$
\begin{aligned}
W_{\mathrm{MSSM}}= & e_{R}^{c T} Y_{\ell} \ell_{L} \cdot H_{d}+d_{R}^{c T} Y_{d} Q_{L} \cdot H_{d} \\
& +u_{R}^{c T} Y_{u} Q_{L} \cdot H_{u}+\mu H_{d} \cdot H_{u} .
\end{aligned}
$$

The SUSY soft-breaking interactions introduce mass terms for the superpartners as well as trilinear couplings between the new sfermions and the Higgs. The relevant terms in our case, not including the quark sector, are:

$$
\begin{aligned}
-\mathcal{L}_{\text {soft }}= & \frac{1}{2}\left(M_{1} \widetilde{B} \widetilde{B}+M_{2} \widetilde{W} \widetilde{W}+M_{3} \tilde{g} \tilde{g}+c . c .\right) \\
& +\tilde{\ell}_{L}^{\dagger} M_{\tilde{\ell}}^{2} \tilde{\ell}_{L}+\tilde{\mathrm{e}}_{R}^{c T} M_{\tilde{\mathrm{e}}}^{2} \tilde{\mathrm{e}}_{R}^{c *}+\tilde{\nu}_{R}^{c T} M_{\tilde{\nu}}^{2} \tilde{\nu}_{R}^{c *} \\
& +\left(\tilde{e}_{R}^{c \dagger} A_{e} \tilde{\ell}_{L} \cdot H_{d}+\tilde{\nu}_{R}^{c \dagger} A_{\nu} \tilde{\ell}_{L} \cdot H_{u}+c . c\right) \\
& +m_{H_{d}}^{2} H_{u}^{*} H_{u}+m_{H_{d}}^{2} H_{d}^{*} H_{d}+\ldots
\end{aligned}
$$

At an energy scale above the heavy $\mathrm{RH} \nu$ mass, $\mu \geq m_{\nu_{3}^{c}}$, an effective operator with the left-handed neutrinos and Higgs fields is generated by the process in figure 1. Integrating out the heavy neutrinos and replacing the Higgs by its vev at low energies, the following mass matrix is produced:

$$
M_{\nu}=-\frac{v_{u}^{2}}{2} Y_{\nu}^{T} M_{R}^{-1} Y_{\nu},
$$

where $v_{u}=v_{h} \sin \beta$ and $v_{h}=246 \mathrm{GeV}$. Neutrino oscillations are directly related to $M_{\nu}$ in eq. (2.8) and provide information about the squared-mass differences and mixing of neutrinos, see table 1. However, those measurements are not enough to fully reconstruct the UV parameters of the model, namely $Y_{\nu}$ and $M_{R}$. 


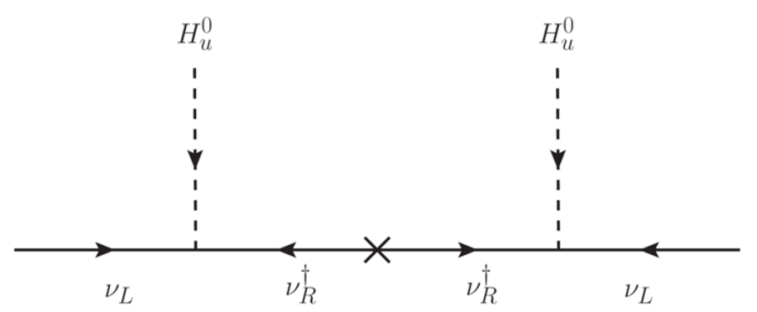

Figure 1. Feynman diagram associated with the type-I seesaw mechanism. The dimension-5 Weinberg operator is effectively generated when the heavy right-handed neutrinos are integrating out.

\begin{tabular}{|ccc|}
\hline Observable & Normal Hierarchy & Inverted Hierarchy \\
\hline$\theta_{12}\left(^{\circ}\right)$ & $33.82_{-0.76}^{+0.78}$ & $33.82_{-0.76}^{+0.78}$ \\
$\theta_{23}\left(^{\circ}\right)$ & $48.3_{-1.9}^{+1.1}$ & $48.6_{-1.5}^{+1.1}$ \\
$\theta_{13}\left(^{\circ}\right)$ & $8.61_{-0.13}^{+0.13}$ & $8.65_{-0.12}^{+0.13}$ \\
$\Delta m_{12}^{2}\left(10^{-5} \mathrm{eV}^{2}\right)$ & $7.39_{-0.20}^{+0.21}$ & $7.39_{-0.20}^{+0.21}$ \\
$\Delta m_{3 \ell}^{2}\left(10^{-3} \mathrm{eV}^{2}\right)$ & $2.523_{-0.030}^{+0.032}$ & $-2.509_{-0.030}^{+0.032}$ \\
\hline
\end{tabular}

Table 1. Global fit on neutrino observables by NuFIT 4.1. (2019) [58]. Similar results have been found in $[59,60]$.

Extensions of the SM may provide additional information through related observables, like CLFV processes. One of the best examples is supersymmetric extensions of the SM supplemented with a type-I seesaw, where slepton soft-breaking masses are related to the neutrino Yukawa couplings. However, additional assumptions are usually made to simplify the analysis of the phenomenology of these models. The minimal strategy consists in presuming universal soft-masses and a concrete structure for the neutrino Yukawa couplings at the GUT scale. Then non-universal entries are generated in the soft masses through the renormalization group evolution (RGE), proportional to the hypothesised Yukawa couplings. It is important to remark that these RGE contributions are always present in SUSY models irrespective of the presence of additional non-universal entries at the GUT scale. Therefore, barring accidental cancellations, these effects are the minimal outcome of supersymmetric seesaw models.

In this framework, we perform the analysis in two limit cases: one in which the rotation angles in $V_{L}^{\nu}$ are small, or CKM-like, and another where the mixing is large, or PMNS-like. These two scenarios should span any intermediate possibility so that general conclusions can be fairly derived. For instance, based on an underlying $\mathrm{SO}(10)$ gauge symmetry, one may consider that $Y_{\nu}$ and $Y_{u}$, in the basis of diagonal Yukawas for charged leptons and down-type 
quarks, are deeply connected at the unification scale through relations such as [61-64]:

$$
\begin{array}{lrl}
\text { - Small Mixing (CKM-like): } & Y_{\nu}^{\mathrm{ckm}}=\mathrm{k}_{\mathrm{GJ}} \mathrm{Y}_{\mathrm{u}} . \\
\text { - Large Mixing (PMNS-like): } & Y_{\nu}^{\mathrm{pmns}} & =\mathrm{k}_{\mathrm{GJ}} \mathrm{Y}_{\mathrm{u}}^{\text {diag }} \mathrm{V}_{\mathrm{pmns}}^{\mathrm{T}}
\end{array}
$$

where, in these equations, we have introduced a Georgi-Jarlskog (GJ) factor, $\mathrm{k}_{\mathrm{GJ}}$, which may arise in some GUT realisations due to the different representations of the unified group that mix to generate the SM Higgs doublet.

Within a $\mathrm{SO}(10)$ model, if the dominant contribution to the Yukawa interactions is due to a Higgs field transforming as a 10, a perfect unification between neutrinos (charged leptons) and up-type (down-type) quarks is expected, so $\mathrm{k}_{\mathrm{GJ}}=1$. Conversely, if the dominant contribution comes from a $\mathbf{1 2 6}$ representation, a factor $\mathrm{k}_{\mathrm{GJ}}=3$ appears between leptons and quarks. Another possibility is having an effective Higgs field transforming as a 120, which may be the product of a $\mathbf{4 5}$ and $\mathbf{1 0}$ representations. The $\mathbf{4 5}$ can acquire a nonzero vev in the flat direction $B-L+\kappa T_{3}$, which preserves the SM and distinguishes between $\mathrm{RH}$ fermions ${ }^{2}[65,66]$. In fact, complete flavour models usually require the combined effect of more than one representation to generate dissimilar hierarchies among generations [6772]. For instance, unification in the down sector as in the classical Georgi-Jarslkog scheme demands $\left|y_{\tau} / y_{b}\right|_{\mathrm{GUT}}=1$ and $\left|y_{\mu} / y_{s}\right|_{\mathrm{GUT}}=\mathrm{k}_{\mathrm{GJ}}=3$. However, nowadays, these relations are no longer favoured phenomenologically [73-76] but the updated range

$$
\left|y_{\mu} / y_{s}\right|_{\mathrm{GUT}}=[2.5,6.5],
$$

obtained in [75]. This is the reason we take to generalize our $\mathrm{k}_{\mathrm{GJ}}$ factor to $\mathrm{k}_{\mathrm{GJ}}=\mathrm{B}-\mathrm{L}+\kappa \mathrm{T}_{3}$. In the case of neutrino Yukawa couplings, we are allowed to consider that the dominant contribution to the up and neutrino Yukawas comes from the representation giving rise to this $\mathrm{k}_{\mathrm{GJ}}$ factor. Then, varying $\kappa$ in the interval where eq. (2.11) is satisfied, one observes that

$$
\left|Y_{\nu}\right|=[0,1 / 2]\left|Y_{u}\right| \text {. }
$$

This is the interval taken for the $\mathrm{k}_{\mathrm{GJ}}$ factor in our numerical analysis.

We explore the parameter space of seesaw NUHM3 models in which some of the stringent conditions of the typical mSUGRA models are relaxed. We introduce three additional degrees of freedom in the scalar soft-breaking sector: instead of one common scalar mass, we will consider the following four

$$
m_{0}^{(1,2)} \neq m_{0}^{(3)} \neq m_{H_{u}} \neq m_{H_{d}}
$$

where we have included a non-universal, but diagonal, charged-slepton mass matrix in the basis of diagonal charged-lepton Yukawa couplings at the GUT scale. The supersymmetric sector of the models is determined by five parameters at the GUT scale,

$$
m_{0}^{(1,2)}, \quad m_{0}^{(3)}, \quad M_{1 / 2}, \quad \tan \beta, \quad A_{0},
$$

and two more at the EW scale,

$$
\mu, \quad M_{A^{0}},
$$

which can be taken in exchange of $m_{H_{u}}$ and $m_{H_{d}}$.

\footnotetext{
${ }^{2} T_{3}$ refers to the third component of a $\mathrm{SU}(2)_{R}$ gauge group which is spontaneously broken afterwards.
} 


\section{RGEs and lepton flavour violation}

The introduction of RH neutrinos makes the effect of the RGEs specially relevant for sleptons [34-37]. During the running, the heavy neutrinos induce off-diagonal entries in the slepton soft terms through radiative corrections. Those flavour-violating interactions allow for CLFV processes that otherwise, within the SM, would be greatly suppressed by the neutrino masses. The main effect occurs for the soft-mass matrices of the LH sleptons and can be worked out by solving the RGEs

$$
\begin{aligned}
\mu \frac{d}{d \mu}\left(M_{\tilde{\ell}_{L}}^{2}\right)= & \mu \frac{d}{d \mu}\left(M_{\tilde{\ell}_{L}}^{2}\right)_{\mathrm{MSSM}} \\
& +\frac{1}{16 \pi^{2}}\left(M_{\tilde{\ell}_{L}}^{2} Y_{\nu}^{\dagger} Y_{\nu}+Y_{\nu}^{\dagger} Y_{\nu} M_{\tilde{\ell}_{L}}^{2}+2\left(Y_{\nu}^{\dagger} M_{\tilde{\nu}}^{2} Y_{\nu}+m_{H_{u}}^{2} Y_{\nu}^{\dagger} Y_{\nu}+A_{\nu}^{\dagger} A_{\nu}\right)\right),
\end{aligned}
$$

where the first term denotes the MSSM contribution in the absence of $\mathrm{RH} \nu$,

$$
\begin{aligned}
\mu \frac{d}{d \mu}\left(M_{\tilde{\ell}_{L}}^{2}\right)_{\mathrm{MSSM}}= & \frac{1}{16 \pi^{2}}\left(M_{\tilde{\ell}_{L}}^{2} Y_{\ell}^{\dagger} Y_{\ell}+Y_{\ell}^{\dagger} Y_{\ell} M_{\tilde{\ell}_{L}}^{2}+2\left(Y_{\ell}^{\dagger} M_{\tilde{\mathrm{e}}}^{2} Y_{\ell}+m_{H_{d}}^{2} Y_{\ell}^{\dagger} Y_{\ell}+A_{\ell}^{\dagger} A_{\ell}\right)\right) \\
& -\mathbb{1}\left(\frac{6}{5} g_{1}^{2}\left|M_{1}\right|^{2}+6 g_{2}^{2}\left|M_{2}\right|^{2}\right)+\mathbb{1} \frac{3}{5} g_{1}^{2} S
\end{aligned}
$$

with $S \equiv \operatorname{Tr}\left[M_{\tilde{Q}_{L}}^{2}+M_{\tilde{d}}^{2}-2 M_{\tilde{u}}^{2}-M_{\tilde{\ell}_{L}}^{2}+M_{\tilde{\mathrm{e}}}^{2}\right]-m_{H_{d}}^{2}+m_{H_{u}}^{2}$. In the basis of diagonal RH $\nu$ and charged-lepton Yukawas, the leading log approximation is proportional to the square of the neutrino Yukawas as:

$$
\left(M_{\tilde{\ell}}^{2}\right)_{i \neq j} \simeq-\frac{2 m_{0}^{2}+m_{H_{u}}^{2}+A_{0}^{2}}{16 \pi^{2}} \sum_{k} Y_{\nu, k i}^{*} Y_{\nu, k j} \log \left(\frac{m_{\mathrm{GUT}}^{2}}{m_{N_{k}}^{2}}\right),
$$

where we take the limit $m_{0}^{(1,2)} \simeq m_{0}^{(3)} \simeq m_{0}$ and approximate $m_{\text {GUT }}$ to be of the order of the scale at which the soft terms appear in the Lagrangian (the typical scale of SUSYbreaking transmission). The main effect, with hierarchical Yukawas, is due to the heaviest Majorana neutrino and happens before its decoupling at $\mu>m_{\nu_{3}^{c}}$. Trilinear couplings receive similar corrections, although they have a smaller impact on the CLFV observables studied here. In contrast, no flavour violation is produced in the $\mathrm{RH}$ charged-slepton sector at one-loop, since the RGEs only depend on $Y_{\ell}$ and on the gauge couplings, hence they are diagonal in the basis where $Y_{\ell}$ is diagonal. The off-diagonal elements produced radiatively enter the total $6 \times 6$ slepton mass matrix as small insertions (compared to the diagonal terms) in the LL and LR/RL sector:

$$
\begin{aligned}
M_{\tilde{\ell}}^{2} & =\left(\begin{array}{cc}
\Delta_{L L} & \Delta_{L R} \\
\Delta_{L R}^{\dagger} & \Delta_{R R}
\end{array}\right) \\
\Delta_{L L} & =M_{\tilde{\ell}_{L}}^{2}+\frac{v_{d}^{2}}{2} Y_{\ell}^{\dagger} Y_{\ell}+\mathbb{1} m_{Z}^{2} \cos 2 \beta\left(-\frac{1}{2}+\sin ^{2} \theta_{w}\right) \\
\Delta_{R R} & =M_{\tilde{\mathrm{e}}_{R}}^{2}+\frac{v_{d}^{2}}{2} Y_{\ell}^{\dagger} Y_{\ell}-\mathbb{1} m_{Z}^{2} \cos 2 \beta \sin ^{2} \theta_{w} \\
\Delta_{L R} & =\frac{v_{d}}{\sqrt{2}}\left(A_{\ell}-\mu^{*} Y_{\ell} \tan \beta\right) .
\end{aligned}
$$




\section{Observables}

Up to now, we have defined the supersymmetric model that we analyse in this project and its RGE evolution to the electroweak scale. The next step will be to compare its predictions with the low-energy observables, to constrain the allowed parameter space or to find possible discrepancies from the SM predictions.

The first observable we have to reproduce is the recently measured value of the Higgs mass, which is a strong constraint on any supersymmetric extension of the SM. Then, as we are mainly interested in the leptonic sector, we concentrate on two main observables: the anomalous magnetic moment of the muon and the radiative process $\mu \rightarrow e \gamma$.

\subsection{Higgs mass}

Previous works have extensively discussed how to accommodate the $125 \mathrm{GeV}$ observed Higgs boson [77, 78] within a minimal supersymmetric framework [79-84]. In the MSSM, it is known that the tree-level value of the lightest Higgs mass is bounded from above by $M_{Z}$ whilst radiative corrections, coming from the fermion-sfermion loops, may increase it up to $135 \mathrm{GeV}$ [85-89]. As those corrections are proportional to the corresponding fermion Yukawa couplings, the dominant contribution is due to the top-stop diagram and can be written as

$$
\Delta m_{h} \simeq \frac{3}{4 \pi^{2}} \cos ^{2} \alpha y_{t}^{2} m_{t}^{2}\left[\ln \left(\frac{m_{\tilde{t}_{1}} m_{\tilde{t}_{2}}}{m_{t}^{2}}\right)+\Delta_{\mathrm{thr}}\right],
$$

where $\alpha$ is the mixing angle between the scalar components of $H_{u}^{0}$ and $H_{d}^{0}$ after EWSB and $\Delta_{\text {thr }}$ stands for the threshold corrections dependent on the stop mixig [90]. While constrained versions of the MSSM, such as mSUGRA, GMSB or AMSB, usually have difficulties to generate the observed mass, scenarios with non-universal conditions at the GUT scale are able to improve their predictions and provide realisations with a low amount of fine-tuning [41, 42, 80].

In the models considered here, the stop mass is determined by the scalar mass parameter $m_{0}^{(3)}$ at the GUT scale. As we will see below, in order to obtain the adequate mass, large values for $m_{0}^{(3)}$ are expected,

$$
m_{0}^{(3)} \gtrsim 4 \mathrm{TeV}
$$

with stops masses in the few-TeV regime,

$$
m_{\tilde{t}_{1}}, m_{\tilde{t}_{2}} \gtrsim 2.5 \mathrm{TeV}
$$

Note that even the most conservative measures of EW fine tuning, $\Delta_{\mathrm{EW}}$, establish that $m_{\tilde{t}_{1}} \lesssim 3 \mathrm{TeV}$ for $\Delta_{\mathrm{EW}}<30[42,91]$ so that the result in eq. (4.3), confirmed by the numerical analysis in section 5, might be in the limit of natural radiative SUSY.

\subsection{Anomalous magnetic moment of the muon}

In the MSSM, leptons receive supersymmetric corrections to their anomalous magnetic moment due to neutralino and chargino loops that effectively generate the dipole operators, 

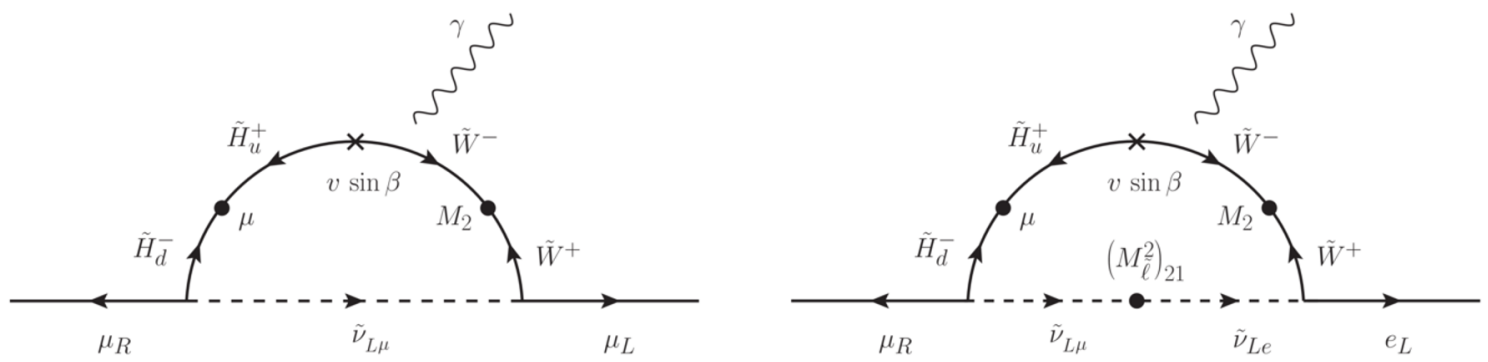

Figure 2. Diagrammatic representation of the leading contribution to $a_{\mu}^{\text {susy }}($ left $)$ and $\operatorname{BR}(\mu \rightarrow \mathrm{e} \gamma)$ (right) in our models.

defined in eq. (B.10) in appendix B.2, where we can find the full expressions, [36, 92],

$$
a_{\ell}^{\text {susy }}=a_{\ell}^{(c)}+a_{\ell}^{(n)} .
$$

The supersymmetric amplitude is usually dominated by the processes where the chirality flip of the fermion occurs at the vertex, which is proportional to the Yukawa coupling and therefore $\tan \beta$-enhanced. The mass insertion approximation (MIA) [93-98] allows us to see this explicitly by means of expanding the full amplitude, extracting the relevant diagrams and identifying the main parameters. It has been implemented in appendix B.2. The diagramatic interpretation of the dominant processes is depicted in figure 2 (left).

In the light higgsino-world scenario, the LSP is the neutralino, which is mainly higgsino and quasi-degenerate in mass with the second-lightest neutralino (NLSP) and the lightest chargino. We observe that the region where $a_{\mu}^{\text {susy }}$ is within the $3 \sigma$ range exhibits the following hierarchies between masses: $\mu \ll M_{2} \lesssim m_{\tilde{\ell}_{L}}$. Then, the process is expected to be dominated by the chargino loop, ${ }^{3}$ since its loop function for $x=\mu^{2} / m_{\tilde{\ell}_{L}}^{2} \ll 1$ is the largest one (see figure 9). Therefore,

$$
a_{\mu}^{\text {susy }} \simeq-\frac{\alpha_{2}}{4 \pi} \frac{m_{\mu}^{2}}{m_{\tilde{\nu}_{\mu}}^{2}} \frac{M_{2} \mu}{M_{2}^{2}-\mu^{2}} F_{2}^{c}\left(x_{2 \tilde{\nu}_{\mu}}, x_{\mu \tilde{\nu}_{\mu}}\right) \tan \beta
$$

where $x_{2 \tilde{\nu}_{\mu}}=M_{2}^{2} / m_{\tilde{\nu}_{\mu}}^{2}, x_{\mu \tilde{\nu}_{\mu}}=\mu^{2} / m_{\tilde{\nu}_{\mu}}^{2}$ and $F_{2}^{c}\left(x_{1}, x_{2}\right) \equiv f_{2}^{(c)}\left(x_{1}\right)-f_{2}^{(c)}\left(x_{2}\right)$ with $f_{2}^{c}(x)$ the loop function provided in appendix A. We compare the exact result worked out by SPheno-4.0.4 [99, 100] versus the MIA expression in figure 3 (left) and notice that the second works quite well for most of the points. Some deviations appear for isolated points in the region where $a_{\mu}^{\text {susy }}$ is very small and contributions from other diagrams may compete and become important.

From eq. (4.5), another phenomenological consequence can be inferred: a SUSY contribution that accounts for the current discrepancy between the experimental and the SM theoretical value of the muon anomalous magnetic moment will require light sneutrinos in the second generation. In the models analysed here, the masses for sfermions of the first

\footnotetext{
${ }^{3}$ More details about the derivation of the chargino and neutralino dominant terms under the MIA can be found in appendix B.2.
} 

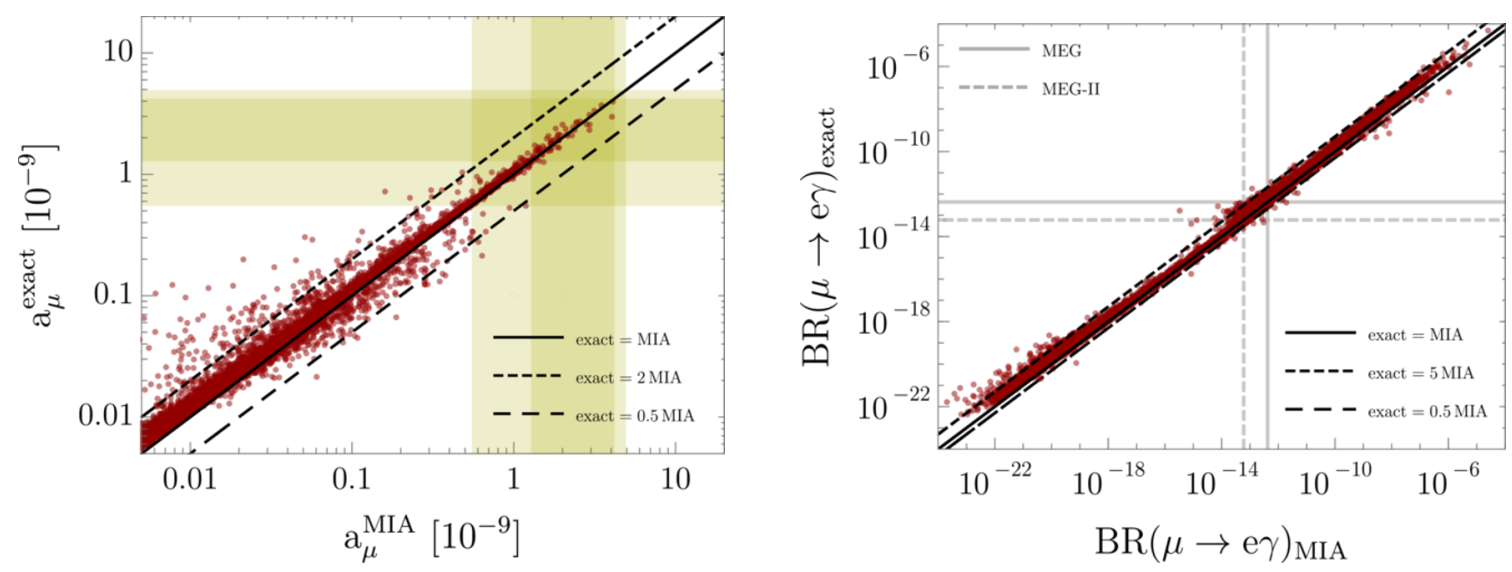

Figure 3. Comparison between the exact values for $a_{\mu}^{\text {susy }}$ (left) and $\mathrm{BR}(\mu \rightarrow \mathrm{e} \gamma)$ (right) computed by SPheno versus the results obtained through the mass insertion approximation in appendix B.2.

\begin{tabular}{|c|c|c|}
\hline LFV Process & Current Limit & Future Limit \\
\hline $\mathrm{BR}(\mu \rightarrow \mathrm{e} \gamma)$ & $4.2 \times 10^{-13}($ MEG at PSI [102]) & $6 \times 10^{-14}($ MEG II $[103])$ \\
\hline $\mathrm{BR}(\mu \rightarrow 3 \mathrm{e})$ & $1.0 \times 10^{-12}($ SINDRUM $[104])$ & $10^{-16}(\mathrm{Mu} 3 \mathrm{e}[105])$ \\
\hline $\mathrm{CR}(\mu-\mathrm{e})_{A_{l}}$ & - & $10^{-17}(\mathrm{Mu} 2 \mathrm{e}[106]$, COMET [105]) \\
\hline $\mathrm{BR}(\tau \rightarrow \mathrm{e} \gamma)$ & $3.3 \times 10^{-8}(\mathrm{BaBar}[107])$ & $5 \times 10^{-9}($ Belle II [108] $)$ \\
\hline $\mathrm{BR}(\tau \rightarrow \mu \gamma)$ & $4.4 \times 10^{-8}(\mathrm{BaBar}[107])$ & $10^{-9}$ (Belle II [108]) \\
\hline $\mathrm{BR}(\tau \rightarrow 3 \mathrm{e})$ & $2.7 \times 10^{-8}($ Belle $[109])$ & $5 \times 10^{-10}($ Belle II [108] $)$ \\
\hline $\operatorname{BR}(\tau \rightarrow 3 \mu)$ & $2.1 \times 10^{-8}($ Belle $[109])$ & $5 \times 10^{-10}($ Belle II [108] $)$ \\
\hline
\end{tabular}

Table 2. Current and future expected limits on CLFV processes.

two generations are determined by the scalar mass $m_{0}^{(1,2)}$ at the GUT scale. Therefore, contrary to $m_{0}^{(3)}$ (see discussion in section 4.1 ), we expect quite small values for $m_{0}^{(1,2)}$ to reproduce $\Delta a_{\mu}$,

$$
m_{0}^{(1,2)} \ll m_{0}^{(3)} .
$$

In practice, eq. (4.6) favours a scalar spectrum where the third generation is heavier than the first two ones. The phenomenology of these models has been previously explored in [101].

\subsection{CLFV observables}

CLFV transitions such as $\ell_{j} \rightarrow \ell_{i} \gamma, \ell_{j} \rightarrow 3 \ell_{i}$ and $\mu \rightarrow$ e in Nuclei are extremely rare in the SM. Actually, we do not expect observing them if the SM is only theory at low energies. The experimental limits on these processes are collected in table 2 .

In the MSSM, the $\ell_{j} \rightarrow \ell_{i}$ transitions are dominated by the dipole operators arising at one-loop level by the exchange of charginos-sneutrinos and neutralinos-charged sleptons, 
eq. (B.10). The flavour switch is feasible due to the flavour-violating interactions generally present in the soft-breaking terms (trilinears and soft masses). Actually, the diagrams for $\ell_{j} \rightarrow \ell_{i}$ and $a_{\ell}^{\text {susy }}$ are essentially identical, except for the flavour transition happening in the former which is absent in the latter. Motivated by this, several articles have evaluated the correlations between $\mu \rightarrow e \gamma$ and $a_{\mu}^{\text {susy }}$ in the MSSM [15, 110-115], although a dedicated discussion in the context of the light higgsino-world scenario is still lacking.

As discussed in section 4.2, in the models under consideration, the lightest neutralinos and chargino are higgsino-like and quasi-degenerate in mass. Assuming that the offdiagonal entries are much smaller than the diagonal ones and applying the MIA for sneutrino and chargino propagators, we find that the branching fraction given by

$$
\frac{\operatorname{BR}(\mu \rightarrow \mathrm{e} \gamma)}{\operatorname{BR}\left(\mu \rightarrow \mathrm{e} \nu_{\mu} \bar{\nu}_{\mathrm{e}}\right)}=\frac{48 \pi^{3} \alpha}{G_{F}^{2}}\left(\left|a_{L}^{\mu \mathrm{e}}\right|^{2}+\left|a_{R}^{\mu \mathrm{e}}\right|^{2}\right)
$$

is well reproduced by the following amplitudes

$$
\begin{aligned}
& a_{R}^{\mu \mathrm{e}} \simeq \frac{\alpha_{2}}{4 \pi} \frac{\left(M_{\tilde{\ell}_{L}}^{2}\right)_{21}}{m_{\tilde{\nu}}^{4}} \frac{M_{2} \mu}{M_{2}^{2}-\mu^{2}} F_{3}^{c}\left(x_{2 \tilde{\nu}}, x_{\mu \tilde{\nu}}\right) \tan \beta, \\
& a_{L}^{\mu \mathrm{e}} \simeq 0,
\end{aligned}
$$

where $F_{3}^{c}\left(x_{2}, x_{\mu}\right) \equiv f_{3}^{(c)}\left(x_{2}\right)-f_{3}^{(c)}\left(x_{\mu}\right)$ and $f_{3}^{(c)}(x)$ the loop function in appendix A. The diagramatic interpretation of eq. (4.8) is very similar to the one for $a_{\mu}^{\text {susy }}$ but with a flavourchanging insertion inside the loop, see figure 2 (right). The accuracy of the approximation is compared to the exact value in figure 3 (right panel). A good agreement is found between both results.

Finally, it is worth commenting the processes $\mu \rightarrow 3 \mathrm{e}$ and $\mu-\mathrm{e}$ conversion. In this case, both receive contributions from penguin diagrams (with Z-boson and photon exchanges), as well as, from box-diagrams. However, again, the $\gamma$-penguin transition (directly connected to $\mu \rightarrow \mathrm{e} \gamma$ ), being $\tan \beta$-enhanced and not suppressed by $M_{Z}$, dominates and the following simple relations hold:

$$
\begin{aligned}
\mathrm{BR}(\mu \rightarrow 3 \mathrm{e}) & \simeq \frac{\alpha}{3 \pi}\left(\log \frac{m_{\mu}^{2}}{m_{e}^{2}}-3\right) \times \mathrm{BR}(\mu \rightarrow \mathrm{e} \gamma) \\
\mathrm{CR}(\mu \mathrm{N} \rightarrow \mathrm{e} \mathrm{N}) & \simeq \alpha \times \mathrm{BR}(\mu \rightarrow \mathrm{e} \gamma) .
\end{aligned}
$$

\section{4 $\operatorname{BR}(\mu \rightarrow \mathrm{e} \gamma)$ versus $a_{\mu}^{\text {susy }}$}

The parallelism between the amplitude in eq. (4.5) and the one in eq. (4.8) is evident. Taking the ratio between them, it is obtained

$$
\frac{a_{R}^{\mu \mathrm{e}}}{a_{\mu}^{\text {susy }}} \simeq \frac{\left(M_{\tilde{\ell}_{L}}^{2}\right)_{21}}{m_{\mu}^{2}} \frac{F_{3}^{c}\left(x_{2 \tilde{\nu}}, x_{\mu \tilde{\nu}}\right)}{F_{2}^{c}\left(x_{2 \tilde{\nu}_{\mu}}, x_{\mu \tilde{\nu}_{\mu}}\right)}
$$



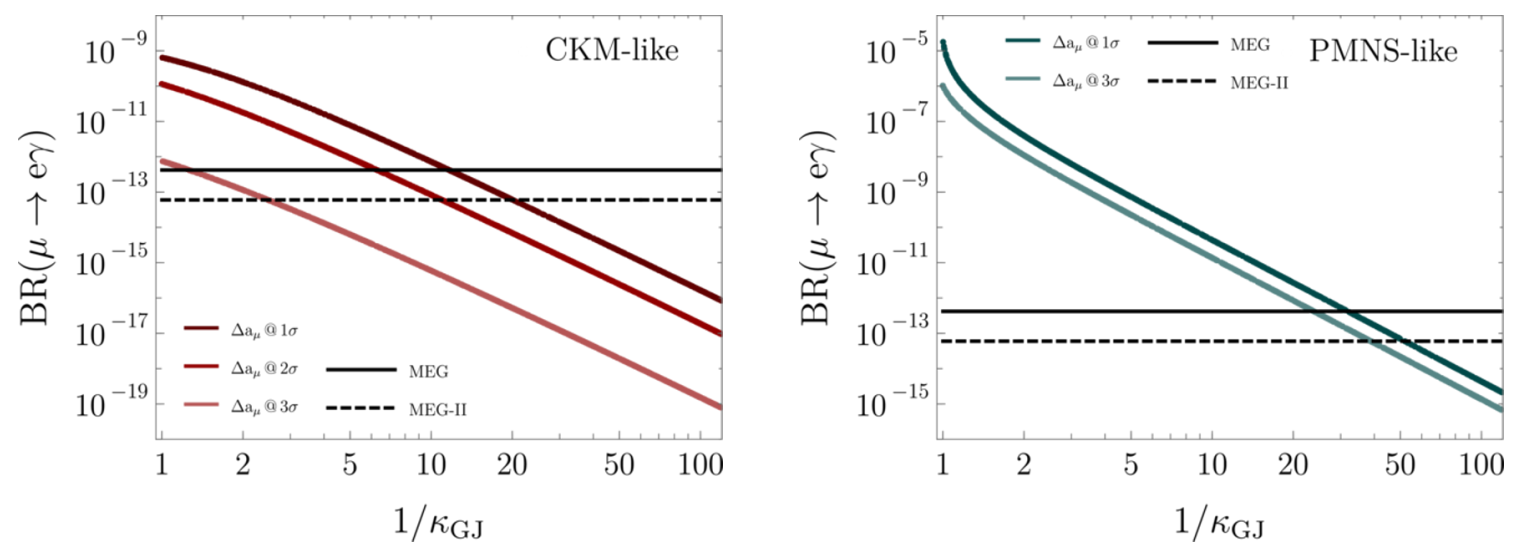

Figure 4. Variation $\operatorname{BR}(\mu \rightarrow \mathrm{e} \gamma)$ with $\mathrm{k}_{\mathrm{GJ}}$ for some representatives points that reproduce $\Delta a_{\mu}$ at different levels of accuracy when $\mathrm{k}_{\mathrm{GJ}}=1$. The black continuous (dashed) line signals the current (future) limit from MEG (MEG-II) [102, 103].

The branching ratio in eq. (4.7) can therefore be rewritten as:

$$
\begin{aligned}
\frac{\operatorname{BR}(\mu \rightarrow \mathrm{e} \gamma)}{\operatorname{BR}\left(\mu \rightarrow \mathrm{e} \nu_{\mu} \bar{\nu}_{\mathrm{e}}\right)} & \simeq \frac{48 \pi^{3} \alpha}{G_{F}^{2} m_{\mu}^{4}}\left|a_{\mu}^{\text {susy }} \frac{\left(M_{\tilde{\ell}_{L}}^{2}\right)_{21}}{m_{\tilde{\nu}}^{2}} \frac{F_{3}^{c}\left(x_{2 \tilde{\nu}}, x_{\mu \tilde{\nu}}\right)}{F_{2}^{c}\left(x_{2 \tilde{\nu}_{\mu}}, x_{\mu \tilde{\nu}_{\mu}}\right)}\right|^{2} \\
& \approx[0.06,4]\left|\frac{a_{\mu}^{\text {susy }}}{10^{-9}} \frac{\left(M_{\tilde{\ell}_{L}}^{2} / m_{\tilde{\nu}}^{2}\right)_{21}}{10^{-4}}\right|^{2} \times 10^{-12}
\end{aligned}
$$

where the interval is due to the variation of the loop functions in the obtained ranges $x_{\mu \tilde{\nu}} \in[0.01,5]$ and $x_{2 \tilde{\nu}} \in[0.1,100]$, see figure 9 (right panel). From eqs. (4.13)-(4.14), one may see that, for those values of $M_{2}, \mu$ and $m_{\tilde{\nu}_{\mu}}$ where $a_{\mu}^{\text {susy }}$ is within the $3 \sigma$ range, an off-diagonal element in the slepton soft-mass matrix around $10^{-4}$ is required to fulfill current and future limits on $\operatorname{BR}(\mu \rightarrow \mathrm{e} \gamma)$. Additionally, eq. (3.5) reveals that the induced off-diagonal term is proportional to the square of the GJ factor introduced in eqs. (2.9)(2.10). Therefore, the absence of any signal in the FC process becomes an indirect upper bound on $\mathrm{k}_{\mathrm{GJ}}$ for our benchmark scenarios. We have explicitly checked the relation between $\mathrm{BR}(\mu \rightarrow \mathrm{e} \gamma)$ and $\mathrm{k}_{\mathrm{GJ}}$ for some representative points. The result is displayed in figure 4 . We select some points that, among other constraints, fulfill $\Delta a_{\mu}$ at different levels of accuracy when $\mathrm{k}_{\mathrm{GJ}}=1$ and evaluate how $\mathrm{BR}(\mu \rightarrow \mathrm{e} \gamma)$ is modified when $\mathrm{k}_{\mathrm{GJ}}$ decreases. As expected, $\operatorname{BR}(\mu \rightarrow \mathrm{e} \gamma)$ is reduced. Consequently, a factor

$$
\mathrm{k}_{\mathrm{GJ}} \simeq 1 / 3(\mathrm{CKM} \text {-like }) \quad \mathrm{k}_{\mathrm{GJ}} \simeq 1 / 50(\text { PMNS-like })
$$

would be needed to conciliate both observables, considering future sensibility from MEG-II. Notice that eq. (4.15) should be taken as a guiding factor, since the RGE effects are not linear in the neutrino Yukawa couplings and the implications on $a_{\mu}^{\text {susy }}$ may change for different values of $\mathrm{k}_{\mathrm{GJ}}$. Then, $\mathrm{k}_{\mathrm{GJ}}$ in eq. (4.15) can change to some extent in the numerical analysis of section 5 . 


\begin{tabular}{|lcc|}
\hline MSSM sector & Range & Sneutrinos \\
\hline$m_{0}^{(1,2)}(\mathrm{TeV})$ & {$[0,15]$} & $m_{\tilde{\nu}_{1,2}}=m_{0}^{(1,2)}$ \\
$m_{0}^{(3)}(\mathrm{TeV})$ & {$[0,15]$} & $m_{\tilde{\nu}_{3}}=m_{0}^{(3)}$ \\
$\tan \beta$ & {$[3,60]$} & \\
$m_{A^{0}}(\mathrm{TeV})$ & {$[0,10]$} & \\
$\mu(\mathrm{TeV})$ & {$[0,1]$} & \\
$M_{1 / 2}(\mathrm{TeV})$ & {$[0,4]$} & \\
$A_{0}$ & {$[-4,4] m_{0}^{(3)}$} & $A_{0}^{\nu}=A_{0}$ \\
\hline
\end{tabular}

Table 3. Parameter space of the numerical scan.

\section{Analysis}

We perform the numerical scan in the ranges shown in table 3. All the parameters are introduced at the GUT scale, except for $\mu$ and $m_{A^{0}}$ that are defined at the EW scale. The running (including neutrinos) is performed with the SPheno-4.0.4 code $[99,100]$ generated by SARAH-4.14.3 [116-118].

Regarding the neutrino sector, once the neutrino Yukawas are known, a phenomenologically viable seesaw mechanism can always be realised by a suitable choice of the righthanded neutrino Majorana mass matrix [61], see appendix C. The running of the neutrinos is performed as detailed in [119]. As a result of the RGE, the neutrino masses and mixing can be slightly modified. To optimize the scan, we feed the program with $Y_{\nu}$ at the GUT scale and the effective mass matrix for the light neutrinos at low energies as inputs.

After the running, the relevant observables are computed and the following constraints are imposed:

- Neutralino LSP and radiative EWSB

- $m_{\chi_{1}^{ \pm}}>160 \mathrm{GeV}[120]$

- $m_{h} \in[122,128] \mathrm{GeV}$

- $m_{\tilde{g}} \geq 2 \mathrm{TeV}$

- $1.12 \times 10^{-9} \leq \mathrm{BR}\left(B_{s} \rightarrow \mu^{+} \mu^{-}\right) \leq 4.48 \times 10^{-9}$

- $2.79 \times 10^{-4} \leq \mathrm{BR}(b \rightarrow s \gamma) \leq 4.63 \times 10^{-4}$

- Neutrino observables within the ranges in table 1.

- Tau rare decays below the current limits in table 2 .

We also check the thermal relic density of the neutralinos. For higgsino-like LSP, the dark matter candidate is a WIMP and the predicted relic abundance is usually below the WMAP measurement [121],

$$
\Omega_{\mathrm{DM}} h^{2}=0.113 \pm 0.0035 \text { at } 68 \% \text {. }
$$



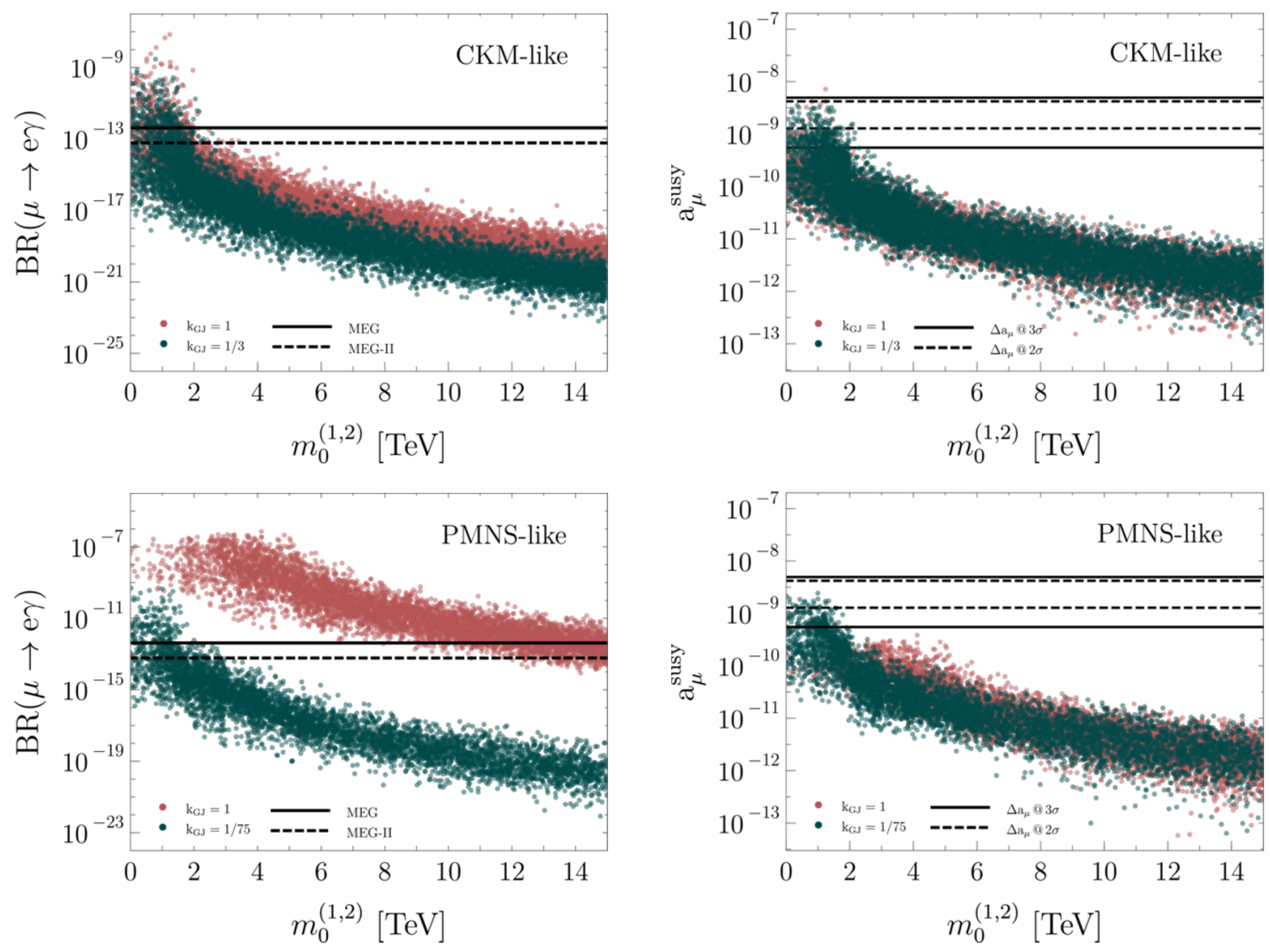

Figure 5. Top: $\operatorname{BR}(\mu \rightarrow \mathrm{e} \gamma)$ (left) and $a_{\mu}^{\text {susy }}$ (right) versus the common soft mass of the first two generations of sleptons at the GUT scale in the CKM-like. Red/light points refer to $\mathrm{k}_{\mathrm{GJ}}=1$ while green/dark points correspond to $\mathrm{k}_{\mathrm{GJ}} \neq 1$. The black (dashed) lines signal the current (future) limit from MEG (MEG-II) [102, 103]. Bottom: the same as above but in the PMNS-like case.

We accept those solutions that predict a lower density and assume that additional effects due to exotic matter, for instance gravitinos, axions, moduli fields or others, can account for the rest $[48,55,122,123]$.

\subsection{Lepton observables}

First, we analyse the dependency of the two transitions, $\operatorname{BR}(\mu \rightarrow \mathrm{e})$ and $a_{\mu}^{\text {susy }}$, on the mass of the sparticle that enter the loop. As discussed in section 4.2 and 4.3, both amplitudes are inversely proportional to the muonic sneutrino mass, see eqs. (4.5) and (4.8). We corroborate this result in figure 5 , where the values of $\operatorname{BR}(\mu \rightarrow \mathrm{e} \gamma)$ and $a_{\mu}^{\text {susy }}$ have been plotted versus $m_{0}^{(1,2)}$, the parameter that controls the mass of the first two generations of sfermions. The first (second) row corresponds to the CKM-like (PMNS-like) case. The left column is for $\operatorname{BR}(\mu \rightarrow \mathrm{e} \gamma)$ and the right column for $a_{\mu}^{\text {susy }}$.

As expected, the largest branching fraction and magnetic moment are observed for $m_{0}^{(1,2)} \lesssim 2 \mathrm{TeV}$. The CKM-like case exhibits, in general, smaller values than the PMNSlike models. This is totally reasonable since the off-diagonal elements of the former are produced by the CKM matrix (almost diagonal) whilst the seconds are dictated by the 

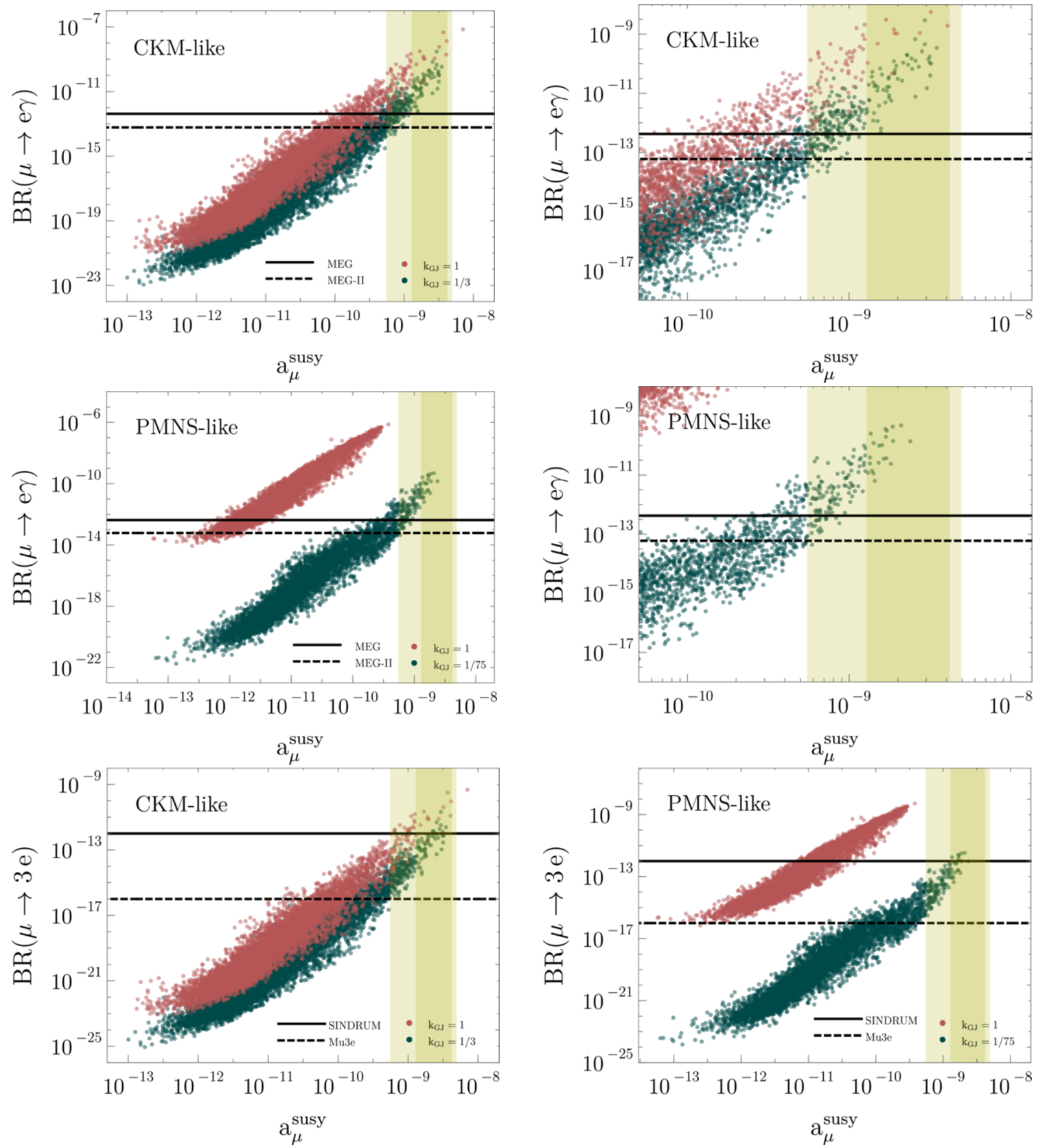

Figure 6. Top: $\operatorname{BR}(\mu \rightarrow \mathrm{e} \gamma)$ versus the supersymmetric contribution to the anomalous magnetic moment of the muon in the CKM-like case. The right panel is a magnification of the region of interest. Red/light points correspond to $\mathrm{k}_{\mathrm{GJ}}=1$ while green/dark points to $\mathrm{k}_{\mathrm{GJ}} \neq 1$. The black (dashed) line signals the current (future) limit from MEG (MEG-II). Middle: the same as before but in the PMNS-like case. Bottom: $\operatorname{BR}(\mu \rightarrow 3 \mathrm{e})$ versus the supersymmetric contribution to the anomalous magnetic moment of the muon in the CKM-like (left) and PMNS-like (right) cases. The black (dashed) line signals the current (future) limit from SINDRUM (Mu3e). 


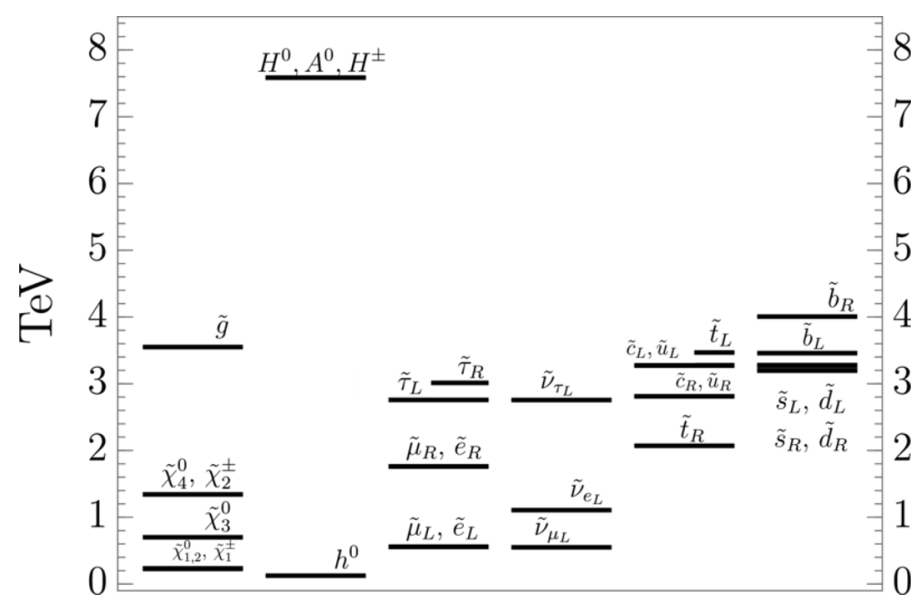

Figure 7. Spectrum for the benchmark point in table 4 .

PMNS matrix with larger mixing angles, see eqs. (2.9)-(2.10). The colours red/light and green/dark refer to $\mathrm{k}_{\mathrm{GJ}}=1$ and $\mathrm{k}_{\mathrm{GJ}} \neq 1$, respectively. We observe that the amplitude for the $\mathrm{FC}$ process decrease when the $\mathrm{k}_{\mathrm{GJ}}$ factor is reduced while, the same variation in $\mathrm{k}_{\mathrm{GJ}}$, has little effect on $a_{\mu}^{\text {susy }}$. This is in agreement with eqs. (4.5), (4.8) and (3.5).

In figure 6 , the results for $\operatorname{BR}(\mu \rightarrow \mathrm{e} \gamma)$ and $a_{\mu}^{\text {susy }}$ are compared. Again, the colour legend is associated with $\mathrm{k}_{\mathrm{GJ}}=1$ (red/light) and $\mathrm{k}_{\mathrm{GJ}} \neq 1$ (green/dark). The first row shows the distribution of points for the CKM-like case. In the left column, the full range of points is represented whereas, in the right column, a magnification of the region of interest can be found. The second row is for the PMNS-like case and shows similar plots. In the case where $\mathrm{k}_{\mathrm{GJ}}=1$, both scenarios are ruled out by the MEG limit, since they predict a larger branching fraction for the process $\mu \rightarrow \mathrm{e} \gamma$. As discussed in section 4.4 , a $\mathrm{k}_{\mathrm{GJ}}$ factor that introduces a splitting between the up-type quarks and neutrinos may reduce the discrepancy and make possible to conciliate all the experimental limits.

For the CKM-like case, the value $\mathrm{k}_{\mathrm{GJ}}=1 / 3$ is enough to fulfill the current bound from MEG and the expected limit from MEG-II. This is in agreement with what was observed in figure 6 . In contrast, the PMNS-like case requires a significantly smaller value, $\mathrm{k}_{\mathrm{GJ}} \sim 1 / 75$. This is consistent with eq. (3.5), which indicates that the generated off-diagonal elements should be larger in the PMNS case, although we obtain a slightly smaller $\mathrm{k}_{\mathrm{GJ}}$ than our estimate in section 4.4. These tiny values of $\mathrm{k}_{\mathrm{GJ}}$ would allow a supersymmetric explanation of the discrepancy with PMNS-like mixings, but they are not natural in a GUT scenario. In eq. (2.10), we are assuming that the representation with a Georgi-Jarlskwithog factor gives the dominant contribution to the neutrino Yukawa couplings. However, a $\mathrm{k}_{\mathrm{GJ}} \sim 1 / 75$ would require all other contributions to be absent or more than two orders of magnitude smaller.

On the other hand, we would like to emphasize that a smaller discrepancy of the experimental results from the SM predictions would enlarge the portion of the parameter space where both observables are consistent. In particular, some points in the CKMlike case with $\mathrm{k}_{\mathrm{GJ}}=1$ may be permitted. This is the case if the discrepancy on the anomalous magnetic moment is reduced due to a slight decrease of the experimental central 


\begin{tabular}{|ccccccc|}
\hline $\boldsymbol{m}_{\mathbf{0}}^{(\mathbf{1}, \mathbf{2})}[\mathrm{TeV}]$ & $\boldsymbol{m}_{\mathbf{0}}^{(\mathbf{3})}[\mathrm{TeV}]$ & $\boldsymbol{M}_{\mathbf{1} \mathbf{2}}[\mathrm{TeV}]$ & $\boldsymbol{A}_{\mathbf{0}}[\mathrm{TeV}]$ & $\boldsymbol{\mu}[\mathrm{TeV}]$ & $\boldsymbol{m}_{\boldsymbol{A}^{\mathbf{0}}}[\mathrm{TeV}]$ & $\boldsymbol{t a n} \boldsymbol{\beta}$ \\
\hline 0.617 & 3.034 & 1.636 & -4.102 & 0.220 & 7.584 & 21.7 \\
\hline
\end{tabular}

Table 4. Input parameters for the benchmark point.

value $[124,125]$ or because of an improvement in the SM calculation in the direction of the measured quantity [16]. ${ }^{4}$

The last row of figure 6 is dedicated to the CLFV decay $\mu \rightarrow 3 \mathrm{e}$. Although the present limit for the process (black, continuous line) is less restrictive that the correspondent to $\mu \rightarrow \mathrm{e} \gamma$, the expected sensitivity (black, dashed line) will become more limiting. Specifically, the region of points where $\mathrm{k}_{\mathrm{GJ}} \neq 1$ and $a_{\mu}^{\text {susy }}$ is compatible with the experimental measurement at $3 \sigma$ or more, will be totally scrutinised. In other words, if the discrepancy in the anomalous magnetic moment of the muon is due to supersymmetric corrections like the ones analysed here, a signal in the $\mu \rightarrow 3 \mathrm{e}$ channel is expected within the $\sim 10^{-12}-10^{-16}$ range. Similar conclusions are extracted from the inspection of the observable $\mathrm{CR}(\mu \mathrm{N} \rightarrow \mathrm{eN})$.

\subsection{Benchmark point}

In this section we discuss the main phenomenological features of a representative benchmark point that satisfies all the constraints detailed before, reproduces $\Delta a_{\mu}$ at $2.5 \sigma$ and is in agreement with MEG limits. The input parameters are presented in table 4, which belong to the CKM-like case. The produced spectrum is depicted in figure 7.

As commented throughout the text, the models analysed here with $\mu \ll M_{1 / 2}$ predict lightest neutralinos and chargino which are higgsino-like and, therefore, almost degenerate in mass. Because of that, the two-body decay of $\tilde{\chi}_{2}^{0}$ and $\tilde{\chi}_{1}^{ \pm}$to $\tilde{\chi}_{1}^{0}$ is forbidden and LHC limits for this compressed spectrum are rather weak. The third neutralino is mostly bino whilst the forth neutralino and second chargino are wino-like. As expected from models with universal gaugino masses at the GUT scale, the gluino is the heaviest superpartner in the gauge sector and, in our case, a mass of $\sim 3.5 \mathrm{TeV}$ is predicted.

The sfermion sector displays the following hierarchy among generations: the lightest states for both sleptons and squarks belong to the first two generations whereas the third generation is associated with the heaviest states. This is consistent with $m_{0}^{(1,2)} \ll m_{0}^{(3)}$. This effect is much stronger in sleptons than in squarks, where the masses are dominated by the gluino mass and the difference in $m_{0}^{(1,2)} \ll m_{0}^{(3)}$ is less relevant. The only exception to this pattern is the lightest stop (a right-handed stop) that turns out to be the lightest squark.

The main effect of the RGE on the sfermion masses is due to the gauge interactions, which increase the mass as they are evolved down to the EW scale, and, mostly for the third generation, the Yukawa couplings and trilinears, which decrease their masses. Thus, in general, heavier left-handed states are expected for the first two generations. For up-type

\footnotetext{
${ }^{4}$ Note, however, that the result from [16] may lead to a deterioration of the EW fit so that new discrepancies with comparable significance would arise for other observables [18].
} 

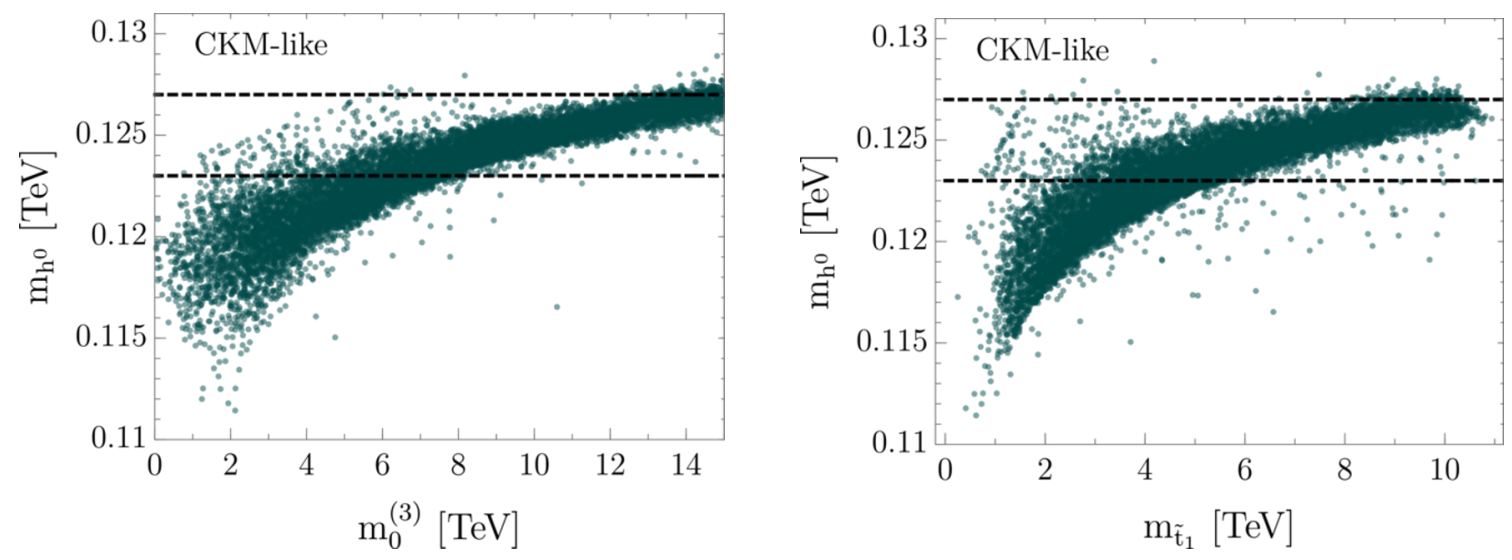

Figure 8. Higgs mass versus the input parameter at the GUT scale $m_{0}^{(3)}$ (left) and the mass of lightest stop at the SUSY scale (right).

squarks, this is also the case in the third generation since the right-handed stop contribution that lowers its mass is proportional to $2 y_{t}^{2}$ while the term associated with the left-handed superpartner goes as $y_{t}^{2}+y_{b}^{2}$. These two effects make the right-handed stop the lightest squark. For down-type squarks, the situation is reversed since the left-handed sbottom receives corrections proportional to $y_{t}^{2}+y_{b}^{2}$ while the right-handed sbottom contribution is $\propto 2 y_{b}^{2}$. This effect is, therefore, opposite to the gauge contribution and, in this particular case, makes the left-handed sbottom lighter than the right-handed one.

The situation is slightly more involved in the slepton sector, where the combined effect of $m_{0}^{(1,2)} \ll m_{0}^{(3)}$ and up-like neutrino Yukawas interfere in the evolution of the left-handed sleptons and make them lighter than the right-handed superpartners, also for the first two generations. This hierarchy is indeed congruent with the explanation of $\Delta a_{\mu}$, since they are the ones mediating the transitions $\mu \rightarrow \mathrm{e} \gamma$ and $\mu \rightarrow \mu \gamma$. Left-handed sneutrinos follow the same pattern than their charged counterpart and satisfy $m_{\tilde{\nu}_{\ell}} \simeq m_{\tilde{\ell}}$.

We check that the predicted spectrum is compatible with LHC direct searches.

Regarding the Higgs sector, we obtain a decoupled spectrum where the lightest Higgs is SM-like and the other CP-even, CP-odd and charged states are much heavier and quasidegenerate in mass. We would like to emphasize that it is possible to reproduce the observed Higgs boson mass and $\Delta a_{\mu}$ simultaneously in these realisations. In figure 8 , we plot the mass of the lightest Higgs boson against $m_{0}^{(3)}$ (left) and the lightest stop mass (right) in the CKM-like case. ${ }^{5}$ Although marginal points that reproduce the Higgs boson mass can be found for lower values of $m_{0}^{(3)}$ and $m_{\tilde{t}_{1}}$, the bulk of the scan indicates that

$$
m_{0}^{(3)} \gtrsim 4 \mathrm{TeV}, \quad m_{\tilde{t}_{1}}, m_{\tilde{t}_{2}} \gtrsim 2.5 \mathrm{TeV}
$$

are preferred to produce a lightest Higgs state near the experimental mass. As noticed before, the result found in eq. (5.2) might be in the limit of radiative natural SUSY which establishes $m_{\tilde{t}_{1}} \lesssim 3 \mathrm{TeV}$ for $\Delta_{\mathrm{EW}}<30[42,91]$.

\footnotetext{
${ }^{5}$ Similar results are found for the PMNS-like case.
} 
In conclusion, $\Delta a_{\mu}$ and the Higgs mass are accomplished simultaneously for nonuniversal models where distinct scalar soft masses are assumed for the first-two and third generation. The lightness of the former allows for a suitable supersymmetric contribution that accounts for the measured discrepancy in the muon anomalous magnetic moment whereas stops within the few-TeV regime are enough to produce a Higgs mass at the observed central value, $125 \mathrm{GeV}$.

\section{Conclusions}

We have investigated how to explain $\Delta a_{\mu}$ in supersymmetric models with right-handed neutrinos while keeping indirect flavour bounds under control.

In section 2, the main ingredients of a supersymmetric type-I seesaw model were presented together with some relations that arise between the Yukawa couplings of different species of sfermions within GUT realisations. In particular, we discussed how a GeorgiJarlskog factor between the Yukawa couplings of up-type quarks and neutrinos may make the latter significantly smaller than the former. We provide an specific example where this happens. As exposed in this section, we focus our analysis on GUT realisations where nonuniversal conditions for the scalar soft masses are assumed at the unification scale. This kind of models are favoured from naturalness considerations since they succeed at reproducing the observed Higgs mass value while producing a low amount of fine-tuning, providing that the Higgs mass parameter is $\mu \lesssim 1 \mathrm{TeV}$. A direct consequence of this condition is that the lightest neutralinos and charginos are higgsino-like and therefore quasi-degenerate in mass. The resulting phenomenology is poorly constrained from direct searches at LHC, so that it remains a highly attractive scenario to explore in the next generation of linear colliders.

In section 3, we recalled a well-known fact: right-handed neutrinos in SUSY models induce off-diagonal elements in the slepton soft mass matrices during the running down to the EW scale through the RGEs. The impact of the these terms in the flavour-changing transitions $\ell_{i} \rightarrow \ell_{j}$ has been investigated in section 4. Analytical expressions have been derived together with a simple relation between the amplitudes of $a_{\mu}^{\text {susy }}$ and $\operatorname{BR}(\mu \rightarrow \mathrm{e} \gamma)$ that holds for our models.

Section 5 was dedicated to our numerical scan. We observe that non-universal models allow for a Higgs boson mass at $125 \mathrm{GeV}$ and a suitable contribution to the anomalous magnetic moment of the muon which can account for the discrepancy between the experimental and the theoretical SM value. For those solutions, charged-lepton flavour-violating processes remain below the experimental limits if the neutrino Yukawas at the GUT scale are suppressed compared to the up-type quark couplings. Due to our little knowledge about the neutrino couplings, this is possible even within a GUT unification context, with a Georgi-Jarlskog factor, $\mathrm{k}_{\mathrm{GJ}}$, as explained in section 2. The required factor between up and neutrino Yukawas depends on the mixing associated with the left-handed neutrinos, small (CKM-like) or large (PMNS-like). While in the first case a $1 / 3$ factor, which can be easily accomodated within a complete theory of flavour, is sufficient, the PMNS-case requires a very small factor, which might be perceived as quite unnatural. Nonetheless, a future change in the experimental results could be favourable for these unified models, if 
it reduces the discrepancy with the SM predictions. Specifically, in this case, a strict unification between the Yukawa couplings of up-type quarks and neutrinos could be reached if the left-handed neutrino Yukawas have a CKM-like mixing.

\section{Acknowledgments}

AM acknowledges support from La-Caixa-Severo Ochoa scholarship. AM and OV are supported by Spanish and European funds under MICIU Grant FPA2017-84543-P and by the "Centro de Excelencia Severo Ochoa" programme under grant SEV-2014-0398. OV acknowledges partial support from the "Generalitat Valenciana" grant PROMETEO2017033. JMY acknowledges funding from the National Natural Science Foundation of China (NNSFC) under grant Nos.11675242, 11821505, and 11851303, from Peng-Huan-Wu Theoretical Physics Innovation Center (11947302), from the CAS Center for Excellence in Particle Physics (CCEPP), from the CAS Key Research Program of Frontier Sciences and from a Key R\&D Program of Ministry of Science and Technology under number 2017YFA0402204. LW acknowledges funding from the National Natural Science Foundation of China (NNSFC) under grant No. 11705093.

\section{A Loop functions}

$$
\begin{aligned}
f_{1}^{(c)}(x) & =\frac{2+3 x-6 x^{2}+x^{3}+6 x \log x}{6(1-x)^{4}} \\
f_{2}^{(c)}(x) & =\frac{-3+4 x-x^{2}-2 \log x}{(1-x)^{3}} \\
f_{3}^{(c)}(x) & =\frac{-5+4 x+x^{2}-2(1+2 x) \log x}{(1-x)^{4}} \\
f_{1}^{(n)}(x) & =\frac{1-6 x+3 x^{2}+2 x^{3}-6 x^{2} \log x}{6(1-x)^{4}} \\
f_{2}^{(n)}(x) & =\frac{1-x^{2}+2 x \log x}{(1-x)^{3}} \\
f_{3}^{(n)}(x) & =\frac{1+4 x-5 x^{2}+2 x(2+x) \log x}{(1-x)^{4}}
\end{aligned}
$$

\section{B Mass insertion approximation}

We follow the conventions in [36] for the neutralino-sfermion-fermion and charginosfermion-fermion couplings:

$$
\begin{aligned}
N_{i, a l}^{L} & =-\frac{g_{2}}{\sqrt{2}}\left[\frac{m_{\ell_{i}}}{m_{W} \cos \beta}\left(O_{N}\right)_{a 3} U_{l i}^{\ell}+2\left(O_{N}\right)_{a 1} \tan \theta_{W} U_{l i+3}^{\ell}\right], \\
N_{i, a l}^{R} & =\frac{g_{2}}{\sqrt{2}}\left[\left(\left(O_{N}\right)_{a 2}+\left(O_{N}\right)_{a 1} \tan \theta_{W}\right) U_{l i}^{\ell}-\frac{m_{\ell_{i}}}{m_{W} \cos \beta}\left(O_{N}\right)_{a 3} U_{l i+3}^{\ell}\right], \\
C_{i, a l}^{L} & =\frac{g_{2} m_{\ell_{i}}}{\sqrt{2} m_{W} \cos \beta}\left(O_{L}\right)_{a 2} U_{l i}^{\nu}, \\
C_{i, a l}^{R} & =-g_{2}\left(O_{R}\right)_{a 1} U_{l i}^{\nu},
\end{aligned}
$$


where $O_{N}$ is the unitary matrix that diagonalises the neutralino mass matrix, $O_{L}$ and $O_{R}$ are the unitary matrices that diagonalise the chargino mass matrix (which, in general, is not hermitian but complex) through a biunitary transformation and $U^{\ell, \nu}$ are the mixing matrices for charged sleptons and sneutrinos.

\section{B.1 Flavour-expansion theorem}

The flavour-expansion theorem [98], on which the mass insertion approximation is based [93-97], states that for any hermitian matrix that is diagonalized by an unitary matrix as

$$
U M^{2} U^{\dagger}=D \equiv \operatorname{Diag}\left(m_{1}^{2}, \ldots, m_{n}^{2}\right),
$$

then any real analytic function of $\mathrm{D}$, which must be analytic around zero and should be possible to express in terms of its McLaurin series, can be expanded as

$$
\left[U^{\dagger} f(D) U\right]_{A B}=\delta_{A B} f\left(M_{A A}^{2}\right)+M_{A B}^{2} \frac{f\left(M_{A A}^{2}\right)-f\left(M_{B B}^{2}\right)}{M_{A A}^{2}-M_{B B}^{2}}+\cdots
$$

For small enough off-diagonal elements in $M^{2}$, the sum in the right-hand side can be fairly good approximated by the first terms. From eq. (B.6), one may also derive the following relation for fermionic amplitudes [98]:

$$
\sum_{i} V_{B i} m_{i} f\left(m_{i}^{2}\right) U_{A i}^{*}=\sum_{C} M_{B C}\left[f\left(M^{\dagger} M\right)\right]_{C A}=\sum_{C}\left[f\left(M M^{\dagger}\right)\right]_{B C} M_{C A},
$$

where $M$ is the mass matrix that is diagonalized by two unitary matrices as

$$
V^{\dagger} M U=D=\operatorname{Diag}\left(m_{1}, \ldots, m_{n}\right)
$$

and

$$
[f(X)]_{A B}=\frac{f\left(X_{A A}\right)-f\left(X_{B B}\right)}{X_{A A}-X_{B B}}
$$

With this prescription, a suitable expression for $a_{\mu}^{\text {susy }}$ and $\operatorname{BR}\left(\ell_{j} \rightarrow \ell_{i} \gamma\right)$ can be computed.

\section{B.2 Expressions for $\operatorname{BR}(\mu \rightarrow \mathrm{e} \gamma)$ and $a_{\mu}^{\text {susy }}$}

By Lorentz invariance, the dipole amplitude for the transition $\ell_{j} \rightarrow \ell_{i} \gamma$ can be written as

$$
\mathcal{M}_{i j}=e m_{\ell_{j}} \varepsilon_{\alpha}(q) \bar{u}_{\ell_{i}}(p-q)\left[i \sigma_{\alpha \beta} q^{\beta}\left(a_{L}^{j i} P_{L}+a_{R}^{j i} P_{R}\right)\right] u_{\ell_{j}}(p) .
$$

The decay rate of the process $\mu \rightarrow \mathrm{e} \gamma$ is then given by

$$
\Gamma(\mu \rightarrow \mathrm{e} \gamma)=\frac{e^{2}}{16 \pi} m_{\mu}^{5}\left(\left|a_{L}^{\mu \mathrm{e}}\right|^{2}+\left|a_{R}^{\mu \mathrm{e}}\right|^{2}\right),
$$

while the anomalous magnetic moment of the muon is described by

$$
a_{\mu} \equiv \frac{(g-2)_{\mu}}{2}=m_{\mu}^{2}\left(a_{L}^{\mu}+a_{R}^{\mu}\right) .
$$


In the MSSM, the amplitudes in eqs. (B.11)-(B.12) contain two terms, one for the sneutrino-chargino loop and another for the charged slepton-neutralino loop

$$
a_{L, R}^{j i}=a_{L, R}^{(n) j i}+a_{L, R}^{(c) j i} .
$$

When $i \neq j$, the amplitudes in eq. (B.11) are

$$
\begin{aligned}
a_{L}^{(n) j i} & =\frac{1}{32 \pi^{2}} \sum_{l, a} \frac{1}{m_{\tilde{\ell}_{l}}^{2}}\left[N_{i, a l}^{L} N_{j, a l}^{L^{*}} f_{1}^{(n)}\left(x_{a l}\right)+\frac{m_{\chi_{a}^{0}}}{m_{\ell_{j}}} N_{i, a l}^{L} N_{j, a l}^{R^{*}} f_{2}^{(n)}\left(x_{a l}\right)\right], \\
a_{L}^{(c) j i} & =-\frac{1}{32 \pi^{2}} \sum_{l, a} \frac{1}{m_{\tilde{\nu}_{l}}^{2}}\left[C_{i, a l}^{L} C_{j, a l}^{L^{*}} f_{1}^{(c)}\left(x_{a l}\right)+\frac{m_{\chi_{a}^{-}}}{m_{\ell_{j}}} C_{i, a l}^{L} C_{j, a l}^{R^{*}} f_{2}^{(c)}\left(x_{a l}\right)\right], \\
a_{R}^{(X) j i} & =\left.a_{L}^{(X) j i}\right|_{L \leftrightarrow R},
\end{aligned}
$$

with $x_{a l}=m_{\chi_{a}^{0}}^{2} / m_{\tilde{\ell}_{l}}^{2}$ in $f_{1,2}^{(n)}(x)$ and $x_{a l}=m_{\chi_{a}^{-}}^{2} / m_{\tilde{\nu}_{l}}^{2}$ in $f_{1,2}^{(c)}(x)$. The loop functions $f_{1,2}^{(c),(n)}(x)$ are compiled in appendix A. If $i=j=2$, the amplitudes in eq. (B.12) are

$$
\begin{aligned}
a_{L}^{(n) \mu} & =-\frac{1}{32 \pi^{2}} \sum_{l, a} \frac{1}{m_{\tilde{\ell}_{l}}^{2}}\left[2 N_{\mu, a l}^{L} N_{\mu, a l}^{L^{*}} f_{1}^{(n)}\left(x_{a l}\right)+\frac{m_{\chi_{a}^{0}}}{m_{\mu}} N_{\mu, a l}^{L} N_{\mu, a l}^{R^{*}} f_{2}^{(n)}\left(x_{a l}\right)\right], \\
a_{L}^{(c) \mu} & =\frac{1}{32 \pi^{2}} \sum_{l, a} \frac{1}{m_{\tilde{\nu}_{l}}^{2}}\left[2 C_{\mu, a l}^{L} C_{\mu, a l}^{L^{*}} f_{1}^{(c)}\left(x_{a l}\right)+\frac{m_{\chi_{a}^{-}}}{m_{\mu}} C_{\mu, a l}^{L} C_{\mu, a l}^{R^{*}} f_{2}^{(c)}\left(x_{a l}\right)\right] \\
a_{R}^{(X) \mu} & =\left.a_{L}^{(X) \mu}\right|_{L \leftrightarrow R} .
\end{aligned}
$$

First, we compute the chargino amplitude. Inspecting eqs. (B.3)-(B.4), one may observe that

$$
\begin{array}{rlrl}
C_{i}^{L} C_{j}^{L} & \propto g_{2}^{2} m_{\ell_{i}} m_{\ell_{j}} & \frac{m_{\chi_{a}^{-}}}{m_{\ell_{j}}} C_{i}^{L} C_{j}^{R} \propto g_{2}^{2} m_{\chi_{a}^{-}} \frac{m_{\ell_{i}}}{m_{\ell_{j}}} \\
C_{i}^{R} C_{j}^{R} \propto g_{2}^{2} & \frac{m_{\chi_{a}^{-}}}{m_{\ell_{j}}} C_{i}^{R} C_{j}^{L} \propto g_{2}^{2} m_{\chi_{a}^{-}} \frac{m_{\ell_{j}}}{m_{\ell_{j}}}
\end{array}
$$

and anticipate that the leading contributions will come from $C_{\mathrm{e}}^{R} C_{\mu}^{L}$ in $\mu \rightarrow \mathrm{e} \gamma$ and from $C_{\mu}^{L} C_{\mu}^{R}$ in $a_{\mu}$. We expand those terms following the MIA guidance. As we are not considering complex phases,

$$
\begin{aligned}
a_{R}^{(c) \mu \mathrm{e}} & \simeq \frac{\alpha_{2}}{4 \pi m_{w} \cos \beta} \sum_{l, a} U_{l \mu}^{\nu}\left(O_{R}\right)_{a 1} \frac{m_{\chi_{a}^{-}}}{m_{\tilde{\nu}_{i}}^{2}} f_{2}^{(c)}\left(x_{a l}\right)\left(O_{L}\right)_{a 2} U_{l \mathrm{e}}^{\nu} \\
& \equiv \frac{\alpha_{2}}{4 \pi m_{w} \cos \beta} \sum_{l} U_{l \mu}^{\nu} F\left(m_{\chi_{a}^{-}}, m_{\tilde{\nu}_{l}}^{2}\right) U_{l \mathrm{e}}^{\nu} \\
a_{\mu}^{(c)} & \simeq-\frac{\alpha_{2} m_{\mu}^{2}}{4 \pi m_{w} \cos \beta} \sum_{l, a} U_{l \mu}^{\nu}\left(O_{R}\right)_{a 1} \frac{m_{\chi_{a}^{-}}}{m_{\tilde{\nu}_{i}}^{2}} f_{2}^{(c)}\left(x_{a l}\right)\left(O_{L}\right)_{a 2} U_{l \mu}^{\nu} \\
& \equiv-\frac{\alpha_{2} m_{\mu}^{2}}{4 \pi m_{w} \cos \beta} \sum_{l} U_{l \mu}^{\nu} F\left(m_{\chi_{a}^{-}}, m_{\tilde{\nu}_{l}}^{2}\right) U_{l \mu}^{\nu}
\end{aligned}
$$


Applying the MIA, eq. (B.6), for the sneutrino propagator and recalling that in our models $m_{\tilde{\nu}_{\mathrm{e}}} \simeq m_{\tilde{\nu}_{\mu}}:$

$$
\begin{aligned}
a_{R}^{(c) \mu \mathrm{e}} & \simeq \frac{\alpha_{2}}{4 \pi m_{w} \cos \beta}\left(M_{\tilde{\ell}_{L}}^{2}\right)_{21}\left[F\left(m_{\chi_{a}^{-}}, m_{\tilde{\nu}_{l}}^{2}\right)\right]_{21} \\
& \approx \frac{\alpha_{2}}{4 \pi m_{w} \cos \beta} \frac{\left(M_{\tilde{\ell}_{L}}^{2}\right)_{21}}{m_{\tilde{\nu}}^{4}} \sum_{a}\left(O_{R}\right)_{a 1} f_{3}^{(c)}\left(x_{a \tilde{\nu}}\right)\left(O_{L}\right)_{a 2} m_{\chi_{a}^{-}}, \\
a_{\mu}^{(c)} & \simeq-\frac{\alpha_{2} m_{\mu}^{2}}{4 \pi m_{w} \cos \beta}\left[F\left(m_{\chi_{a}^{-}}, m_{\tilde{\nu}_{l}}^{2}\right)\right]_{22} \\
& \approx-\frac{\alpha_{2}}{4 \pi m_{w} \cos \beta} \frac{m_{\mu}^{2}}{m_{\tilde{\nu}_{\mu}}^{2}} \sum_{a}\left(O_{R}\right)_{a 1} f_{2}^{c}\left(x_{a \tilde{\nu}_{\mu}}\right)\left(O_{L}\right)_{a 2} m_{\chi_{a}^{-}},
\end{aligned}
$$

with $f_{3}^{(c)}(x) \equiv f_{2}^{(c)}(x)+x f_{2}^{(c)}{ }^{\prime}(x)$ explicitly given in appendix A. We repeat the same procedure for the chargino propagator taking into account the derived equality in eq. (B.7). Then,

$$
\begin{aligned}
a_{R}^{(c) \mu \mathrm{e}} & \simeq \frac{\alpha_{2}}{4 \pi m_{w} \cos \beta} \frac{\left(M_{\tilde{\ell}_{L}}^{2}\right)_{21}}{m_{\tilde{\nu}}^{4}} \sum_{a}\left(M_{C}\right)_{1 a}\left[f_{3}^{(c)}\left(x_{a \tilde{\nu}_{\mu}}\right)\right]_{a 2} \\
& \approx \frac{\alpha_{2}}{4 \pi} \frac{\left(M_{\tilde{\ell}_{L}}^{2}\right)_{21}}{m_{\tilde{\nu}}^{4}} \frac{M_{2} \mu}{M_{2}^{2}-\mu^{2}} F_{3}^{c}\left(x_{2 \tilde{\nu}}, x_{\mu \tilde{\nu})} \tan \beta .\right. \\
a_{\mu}^{(c)} & \simeq-\frac{\alpha_{2}}{4 \pi m_{w} \cos \beta} \frac{m_{\mu}^{2}}{m_{\tilde{\nu}_{\mu}}^{2}} \sum_{a}\left(M_{C}\right)_{1 a}\left[f_{2}^{(c)}\left(x_{a \tilde{\nu}_{\mu}}\right)\right]_{a 2} \\
& \approx-\frac{\alpha_{2}}{4 \pi} \frac{m_{\mu}^{2}}{m_{\tilde{\nu}_{\mu}}^{2}} \frac{M_{2} \mu}{M_{2}^{2}-\mu^{2}} F_{2}^{c}\left(x_{2 \tilde{\nu}_{\mu}}, x_{\left.\mu \tilde{\nu}_{\mu}\right)} \tan \beta .\right.
\end{aligned}
$$

where in the last equalities we have neglected those terms proportional to $\cos \beta$. The functions $F_{X}^{c}\left(x_{1}, x_{2}\right)$ are defined as $F_{X}^{c}\left(x_{1}, x_{2}\right) \equiv f_{X}^{(c)}\left(x_{1}\right)-f_{X}^{(c)}\left(x_{2}\right)$.

We can repeat the same procedure for the neutralino amplitude. From eqs. (B.1)-(B.2) and eqs. (B.14) and (B.17), we observe that the dominant term for $\mu \rightarrow \mathrm{e} \gamma$ comes from the combination $N_{\mu}^{L} N_{\mathrm{e}}^{R}$ whereas, in the case of $a_{\mu}$, it is produced by $N_{\mu}^{L} N_{\mu}^{R}$. Then, following the same steps than for the chargino loop, one would obtain:

$$
\begin{aligned}
a_{R}^{(n) \mu \mathrm{e}} & \simeq-\frac{\alpha_{2}}{8 \pi} \frac{\left(M_{\tilde{\ell}_{L}}^{2}\right)_{21}}{m_{\tilde{\nu}_{\mu}}^{4}} \frac{M_{2} \mu}{M_{2}^{2}-\mu^{2}} F_{3}^{n}\left(x_{2 \tilde{\nu}_{\mu}}, x_{\mu \tilde{\nu}_{\mu}}\right) \tan \beta \\
a_{\mu}^{(n)} & \simeq-\frac{\alpha_{2}}{8 \pi} \frac{m_{\mu}^{2}}{m_{\tilde{\mu}}^{2}} \frac{M_{2} \mu}{M_{2}^{2}-\mu^{2}} F_{2}^{n}\left(x_{2 \tilde{\nu}_{\mu}}, x_{\mu \tilde{\nu}_{\mu}}\right) \tan \beta
\end{aligned}
$$

with $F_{X}^{n}\left(x_{1}, x_{2}\right)=f_{X}^{(n)}\left(x_{1}\right)-f_{X}^{(n)}\left(x_{2}\right)$ and $f_{3}^{(n)} \equiv f_{2}^{(n)}(x)+x f_{2}^{(n)^{\prime}}(x)$ explicitely given in appendix A. The result is identical to the chargino contribution, except for an additional $1 / 2$ factor and the loop functions involved. In a generic model where neutralinos and charginos have similar masses, both amplitudes are equally important. 

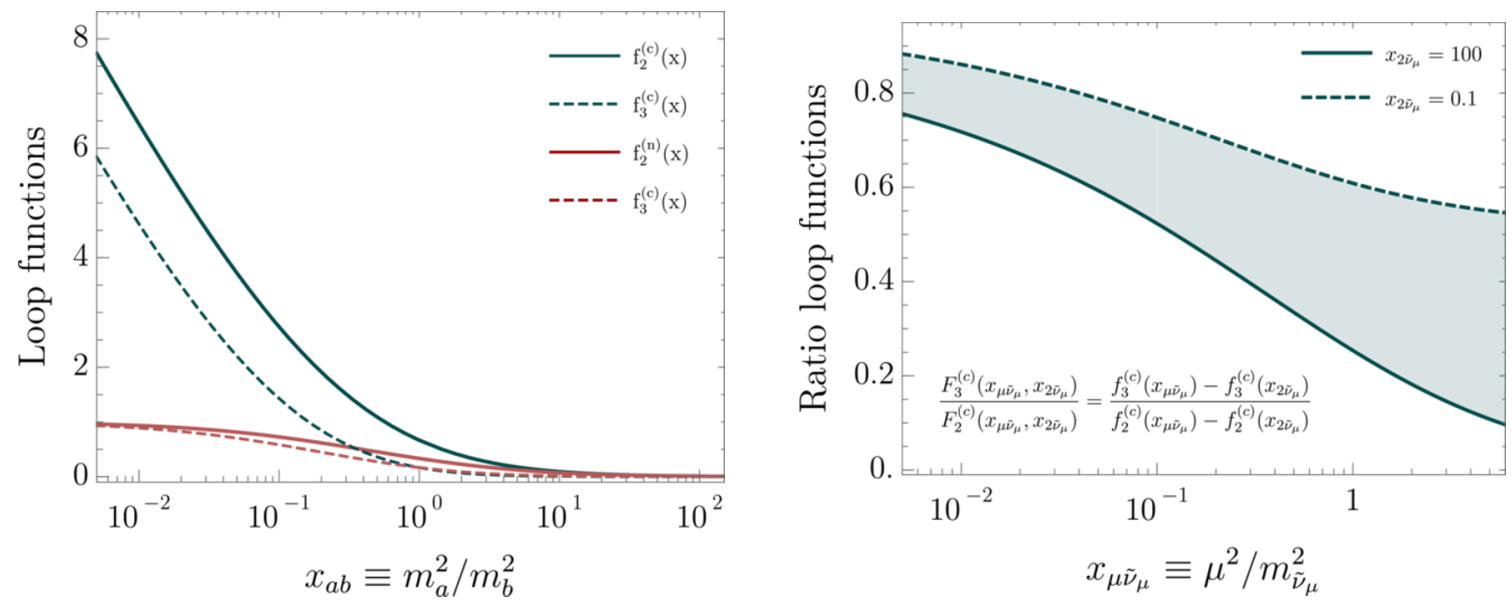

Figure 9. Loop functions involved in $a_{\mu}^{\text {susy }}$ (solid line) and $\operatorname{BR}(\mu \rightarrow \mathrm{e})$ (dashed) corresponding to charginos (green/dark) and neutralinos (red/light).

In the models considered here, however, we notice the following hierarchies between masses in the region where $a_{\mu}^{\text {susy }}$ is within the $3 \sigma$ range: $\mu \ll M_{2} \lesssim m_{\tilde{\mu}}, m_{\tilde{\nu}_{\mu}}$. Evaluating the chargino and neutralino loop functions, figure 9 (left panel), one may see that the former dominates for $x=\mu^{2} / m_{\tilde{\nu}_{\mu}}^{2} \ll 1$. Therefore, we expect that the total amplitudes are governed by the chargino terms given in eqs. (B.30)-(B.31). In figure 3, we compared the approximate expressions against the exact results obtained with SPheno. Both cases display a good agreement for the region of interest. Deviations from the exact result are observed only for small values of $a_{\mu}$ and the region of large $\operatorname{BR}(\mu \rightarrow \mathrm{e} \gamma)$, where other loops may compete and become important.

\section{Majorana mass matrix}

Here we detail how the Majorana mass matrix can be extracted once the neutrino Dirac matrix is fixed. In the CKM-like case, the neutrino Yukawa matrix is symmetric and can be expressed as:

$$
Y_{\nu}^{\mathrm{ckm}}=Y_{u}=V_{\mathrm{ckm}}^{T} Y_{u}^{\mathrm{diag}} V_{\mathrm{ckm}} .
$$

A $M_{R}$ mass matrix that correctly reproduces the light neutrino masses and mixing can be inferred from eq. (2.8) as:

$$
M_{R}=v_{u}^{2} Y_{\nu}^{\mathrm{ckm}}\left(V_{\mathrm{pmns}} m_{\nu}^{\mathrm{diag}^{-1}} V_{\mathrm{pmns}}^{T}\right) Y_{\nu}^{\mathrm{ckm}}
$$

with $Y_{\nu}^{\mathrm{ckm}}$ fixed by eq. (C.1). In the PMNS-like case, the neutrino Yukawa matrix is given by:

$$
Y_{\nu}^{\mathrm{pmns}}=Y_{u}^{\operatorname{diag}} V_{\mathrm{pmns}}^{T}
$$

The $M_{R}$ mass matrix that automatically satisfies all neutrino observables is diagonal and it is simply given by:

$$
M_{R}=v_{u}^{2} Y_{u}^{\text {diag }} m_{\nu}^{\text {diag }^{-1}} Y_{u}^{\text {diag }},
$$


with eigenvalues

$$
M_{R}=\operatorname{Diag}\left(\frac{m_{u}^{2}}{m_{\nu_{1}}}, \frac{m_{c}^{2}}{m_{\nu_{2}}}, \frac{m_{t}^{2}}{m_{\nu_{3}}}\right) .
$$

Open Access. This article is distributed under the terms of the Creative Commons Attribution License (CC-BY 4.0), which permits any use, distribution and reproduction in any medium, provided the original author(s) and source are credited.

\section{References}

[1] Super-Kamiokande collaboration, Evidence for oscillation of atmospheric neutrinos, Phys. Rev. Lett. 81 (1998) 1562 [hep-ex/9807003] [INSPIRE].

[2] Super-Kamiokande collaboration, Study of the atmospheric neutrino flux in the multi-GeV energy range, Phys. Lett. B 436 (1998) 33 [hep-ex/9805006] [INSPIRE].

[3] Muon G-2 collaboration, Final report of the muon E821 anomalous magnetic moment measurement at BNL, Phys. Rev. D 73 (2006) 072003 [hep-ex/0602035] [INSPIRE].

[4] RBC and UKQCD collaborations, Calculation of the hadronic vacuum polarization contribution to the muon anomalous magnetic moment, Phys. Rev. Lett. 121 (2018) 022003 [arXiv: 1801.07224] [INSPIRE].

[5] A. Keshavarzi, D. Nomura and T. Teubner, Muon g- 2 and $\alpha\left(M_{Z}^{2}\right)$ : a new data-based analysis, Phys. Rev. D 97 (2018) 114025 [arXiv:1802.02995] [INSPIRE].

[6] M. Davier, A. Hoecker, B. Malaescu and Z. Zhang, A new evaluation of the hadronic vacuum polarisation contributions to the muon anomalous magnetic moment and to $\alpha\left(m_{Z}^{2}\right)$, Eur. Phys. J. C 80 (2020) 241 [arXiv: 1908.00921] [InSPIRE].

[7] M. Davier, A. Hoecker, B. Malaescu and Z. Zhang, Reevaluation of the hadronic vacuum polarisation contributions to the Standard Model predictions of the muon $g-2$ and $\alpha\left(m_{Z}^{2}\right)$ using newest hadronic cross-section data, Eur. Phys. J. C 77 (2017) 827 [arXiv: 1706. 09436] [INSPIRE].

[8] M. Davier, A. Hoecker, B. Malaescu and Z. Zhang, Reevaluation of the hadronic contributions to the muon $g-2$ and to $\alpha\left(m_{Z}^{2}\right)$, Eur. Phys. J. C 71 (2011) 1515 [Erratum ibid. C 72 (2012) 1874] [arXiv:1010.4180] [INSPIRE].

[9] M. Endo, K. Hamaguchi, S. Iwamoto and T. Kitahara, Muon g- 2 vs LHC run 2 in supersymmetric models, JHEP 04 (2020) 165 [arXiv:2001.11025] [INSPIRE].

[10] M. Endo and W. Yin, Explaining electron and muon $g-2$ anomaly in SUSY without lepton-flavor mixings, JHEP 08 (2019) 122 [arXiv:1906.08768] [INSPIRE].

[11] M. Abdughani, K.-I. Hikasa, L. Wu, J.M. Yang and J. Zhao, Testing electroweak SUSY for muon $g-2$ and dark matter at the LHC and beyond, JHEP 11 (2019) 095 [arXiv: 1909.07792] [INSPIRE].

[12] M. Badziak and K. Sakurai, Explanation of electron and muon $g-2$ anomalies in the MSSM, JHEP 10 (2019) 024 [arXiv: 1908.03607] [INSPIRE].

[13] P. Cox, C. Han and T.T. Yanagida, Muon $g-2$ and dark matter in the minimal supersymmetric Standard Model, Phys. Rev. D 98 (2018) 055015 [arXiv:1805.02802] [INSPIRE]. 
[14] P. Cox, C. Han, T.T. Yanagida and N. Yokozaki, Gaugino mediation scenarios for muon $g-2$ and dark matter, JHEP 08 (2019) 097 [arXiv: 1811.12699] [INSPIRE].

[15] M. Lindner, M. Platscher and F.S. Queiroz, A call for new physics: the muon anomalous magnetic moment and lepton flavor violation, Phys. Rept. 731 (2018) 1 [arXiv: 1610.06587] [INSPIRE].

[16] S. Borsányi et al., Leading-order hadronic vacuum polarization contribution to the muon magnetic moment from lattice QCD, arXiv:2002.12347 [INSPIRE].

[17] M. Passera, W.J. Marciano and A. Sirlin, The muon $g-2$ and the bounds on the Higgs boson mass, Phys. Rev. D 78 (2008) 013009 [arXiv:0804.1142] [INSPIRE].

[18] A. Crivellin, M. Hoferichter, C.A. Manzari and M. Montull, Hadronic vacuum polarization: $(g-2)_{\mu}$ versus global electroweak fits, arXiv:2003.04886 [INSPIRE].

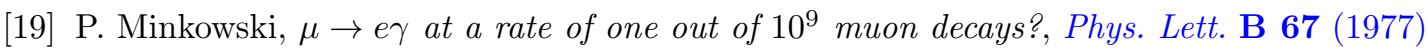
421 [INSPIRE].

[20] T. Yanagida, Horizontal gauge symmetry and masses of neutrinos, Conf. Proc. C 7902131 (1979) 95 [INSPIRE].

[21] R.N. Mohapatra and G. Senjanović, Neutrino mass and spontaneous parity nonconservation, Phys. Rev. Lett. 44 (1980) 912 [INSPIRE].

[22] M. Gell-Mann, P. Ramond and R. Slansky, Complex spinors and unified theories, Conf. Proc. C 790927 (1979) 315 [arXiv: 1306.4669] [InSPIRE].

[23] S.L. Glashow, The future of elementary particle physics, in Quarks and leptons, M. Lévy et al. eds., Springer, Boston, MA, U.S.A. (1980), pg. 687 [InSPIRE].

[24] J. Schechter and J.W.F. Valle, Neutrino masses in $\mathrm{SU}(2) \times \mathrm{U}(1)$ theories, Phys. Rev. D 22 (1980) 2227 [INSPIRE].

[25] S. Weinberg, Baryon and lepton nonconserving processes, Phys. Rev. Lett. 43 (1979) 1566 [INSPIRE].

[26] K.G. Wilson, The renormalization group and strong interactions, Phys. Rev. D 3 (1971) 1818 [INSPIRE].

[27] G. 't Hooft et al., Recent developments in gauge theories, in Proceedings, NATO Advanced Study Institute, Cargese, France, 26 August-8 September 1979, NATO Sci. Ser. B 59 (1980) 1 [INSPIRE].

[28] H. Goldberg, Constraint on the photino mass from cosmology, Phys. Rev. Lett. 50 (1983) 1419 [Erratum ibid. 103 (2009) 099905] [INSPIRE].

[29] J.R. Ellis, J.S. Hagelin, D.V. Nanopoulos, K.A. Olive and M. Srednicki, Supersymmetric relics from the big bang, Nucl. Phys. B 238 (1984) 453 [INSPIRE].

[30] S. Dimopoulos, S. Raby and F. Wilczek, Supersymmetry and the scale of unification, Phys. Rev. D 24 (1981) 1681 [INSPIRE].

[31] J.R. Ellis, S. Kelley and D.V. Nanopoulos, Probing the desert using gauge coupling unification, Phys. Lett. B 260 (1991) 131 [INSPIRE].

[32] U. Amaldi, W. de Boer and H. Furstenau, Comparison of grand unified theories with electroweak and strong coupling constants measured at LEP, Phys. Lett. B 260 (1991) 447 [INSPIRE]. 
[33] P. Langacker and M.-X. Luo, Implications of precision electroweak experiments for $M_{t}, \rho_{0}$, $\sin ^{2} \theta_{W}$ and grand unification, Phys. Rev. D 44 (1991) 817 [InSPIRE].

[34] F. Borzumati and A. Masiero, Large muon and electron number violations in supergravity theories, Phys. Rev. Lett. 57 (1986) 961 [INSPIRE].

[35] A. Ilakovac and A. Pilaftsis, Flavor violating charged lepton decays in seesaw-type models, Nucl. Phys. B 437 (1995) 491 [hep-ph/9403398] [INSPIRE].

[36] J. Hisano, T. Moroi, K. Tobe and M. Yamaguchi, Lepton flavor violation via right-handed neutrino Yukawa couplings in supersymmetric Standard Model, Phys. Rev. D 53 (1996) 2442 [hep-ph/9510309] [INSPIRE].

[37] J.A. Casas and A. Ibarra, Oscillating neutrinos and $\mu \rightarrow e, \gamma$, Nucl. Phys. B 618 (2001) 171 [hep-ph/0103065] [INSPIRE].

[38] K.L. Chan, U. Chattopadhyay and P. Nath, Naturalness, weak scale supersymmetry and the prospect for the observation of supersymmetry at the Tevatron and at the CERN LHC, Phys. Rev. D 58 (1998) 096004 [hep-ph/9710473] [INSPIRE].

[39] J.L. Feng, K.T. Matchev and T. Moroi, Multi-TeV scalars are natural in minimal supergravity, Phys. Rev. Lett. 84 (2000) 2322 [hep-ph/9908309] [INSPIRE].

[40] J.L. Feng, K.T. Matchev and T. Moroi, Focus points and naturalness in supersymmetry, Phys. Rev. D 61 (2000) 075005 [hep-ph/9909334] [INSPIRE].

[41] H. Baer, V. Barger, P. Huang, A. Mustafayev and X. Tata, Radiative natural SUSY with a 125 GeV Higgs boson, Phys. Rev. Lett. 109 (2012) 161802 [arXiv:1207.3343] [INSPIRE].

[42] H. Baer, V. Barger, P. Huang, D. Mickelson, A. Mustafayev and X. Tata, Radiative natural supersymmetry: reconciling electroweak fine-tuning and the Higgs boson mass, Phys. Rev. D 87 (2013) 115028 [arXiv: 1212.2655] [INSPIRE].

[43] P. Nath and R.L. Arnowitt, Nonuniversal soft SUSY breaking and dark matter, Phys. Rev. D 56 (1997) 2820 [hep-ph/9701301] [inSPIRE].

[44] A. Lleyda and C. Muñoz, Nonuniversal soft scalar masses in supersymmetric theories, Phys. Lett. B 317 (1993) 82 [hep-ph/9308208] [INSPIRE].

[45] T. Kobayashi, D. Suematsu and Y. Yamagishi, Gauge coupling unification due to nonuniversal soft supersymmetry breaking, Phys. Lett. B 329 (1994) 27 [hep-ph/9403330] [INSPIRE].

[46] G.L. Kane and J.D. Wells, Higgsino cold dark matter motivated by collider data, Phys. Rev. Lett. 76 (1996) 4458 [hep-ph/9603336] [INSPIRE].

[47] G. Kane, Sphenomenology - an overview, with a focus on a higgsino LSP world, and on eventual tests of string theory, Nucl. Phys. Proc. Suppl. B 62 (1998) 144.

[48] H. Baer, V. Barger and P. Huang, Hidden SUSY at the LHC: the light higgsino-world scenario and the role of a lepton collider, JHEP 11 (2011) 031 [arXiv:1107.5581] [INSPIRE].

[49] C. Han, A. Kobakhidze, N. Liu, A. Saavedra, L. Wu and J.M. Yang, Probing light higgsinos in natural SUSY from monojet signals at the LHC, JHEP 02 (2014) 049 [arXiv:1310.4274] [INSPIRE].

[50] M. Abdughani, L. Wu and J.M. Yang, Status and prospects of light bino-higgsino dark matter in natural SUSY, Eur. Phys. J. C 78 (2018) 4 [arXiv:1705.09164] [INSPIRE]. 
[51] J. Ren, L. Wu, J.M. Yang and J. Zhao, Exploring supersymmetry with machine learning, Nucl. Phys. B 943 (2019) 114613 [arXiv:1708.06615] [InSPIRE].

[52] N. Liu and L. Wu, An indirect probe of the higgsino world at the CEPC, Eur. Phys. J. C 77 (2017) 868 [arXiv: 1705. 02534] [inSPIRE].

[53] C. Han, R. Li, R.-Q. Pan and K. Wang, Searching for the light Higgsinos in the MSSM at future e-p colliders, Phys. Rev. D 98 (2018) 115003 [arXiv: 1802.03679] [INSPIRE].

[54] M. Abdughani, J. Ren, L. Wu, J.M. Yang and J. Zhao, Supervised deep learning in high energy phenomenology: a mini review, Commun. Theor. Phys. 71 (2019) 955 [arXiv: 1905. 06047] [INSPIRE].

[55] C. Han, Higgsino dark matter in a non-standard history of the universe, Phys. Lett. B 798 (2019) 134997 [arXiv: 1907.09235] [INSPIRE].

[56] B.P. Padley, K. Sinha and K. Wang, Natural supersymmetry, muon $g-2$ and the last crevices for the top squark, Phys. Rev. D 92 (2015) 055025 [arXiv:1505.05877] [InSPIRE].

[57] H. Baer, V. Barger and H. Serce, Lepton flavor violation from SUSY with non-universal scalars, Phys. Rev. Research. 1 (2019) 033022 [arXiv:1907.06693] [INSPIRE].

[58] I. Esteban, M.C. Gonzalez-Garcia, A. Hernandez-Cabezudo, M. Maltoni and T. Schwetz, Global analysis of three-flavour neutrino oscillations: synergies and tensions in the determination of $\theta_{23}, \delta_{C P}$ and the mass ordering, JHEP 01 (2019) 106 [arXiv: 1811.05487] [INSPIRE].

[59] P.F. de Salas, D.V. Forero, C.A. Ternes, M. Tortola and J.W.F. Valle, Status of neutrino oscillations 2018: $3 \sigma$ hint for normal mass ordering and improved CP sensitivity, Phys. Lett. B 782 (2018) 633 [arXiv:1708.01186] [INSPIRE].

[60] F. Capozzi, E. Di Valentino, E. Lisi, A. Marrone, A. Melchiorri and A. Palazzo, Global constraints on absolute neutrino masses and their ordering, Phys. Rev. D 95 (2017) 096014 [arXiv: 1703.04471] [INSPIRE].

[61] A. Masiero, S.K. Vempati and O. Vives, Seesaw and lepton flavor violation in SUSY SO(10), Nucl. Phys. B 649 (2003) 189 [hep-ph/0209303] [INSPIRE].

[62] L. Calibbi, A. Faccia, A. Masiero and S.K. Vempati, Lepton flavour violation from SUSY-GUTs: where do we stand for MEG, PRISM/PRIME and a super flavour factory, Phys. Rev. D 74 (2006) 116002 [hep-ph/0605139] [INSPIRE].

[63] L. Calibbi, R.N. Hodgkinson, J. Jones Perez, A. Masiero and O. Vives, Flavour and collider interplay for SUSY at LHC7, Eur. Phys. J. C 72 (2012) 1863 [arXiv:1111.0176] [InSPIRE].

[64] L. Calibbi, D. Chowdhury, A. Masiero, K.M. Patel and S.K. Vempati, Status of supersymmetric type-I seesaw in $\mathrm{SO}(10)$ inspired models, JHEP 11 (2012) 040 [arXiv: 1207.7227] [INSPIRE].

[65] G.G. Ross and L. Velasco-Sevilla, Symmetries and fermion masses, Nucl. Phys. B 653 (2003) 3 [hep-ph/0208218] [INSPIRE].

[66] S.F. King and G.G. Ross, Fermion masses and mixing angles from SU(3) family symmetry and unification, Phys. Lett. B 574 (2003) 239 [hep-ph/0307190] [INSPIRE].

[67] G.G. Ross, L. Velasco-Sevilla and O. Vives, Spontaneous CP-violation and non-Abelian family symmetry in SUSY, Nucl. Phys. B 692 (2004) 50 [hep-ph/0401064] [INSPIRE]. 
[68] D. Das, M.L. López-Ibáñez, M.J. Pérez and O. Vives, Effective theories of flavor and the nonuniversal MSSM, Phys. Rev. D 95 (2017) 035001 [arXiv:1607.06827] [INSPIRE].

[69] I. de Medeiros Varzielas and G.G. Ross, SU(3) family symmetry and neutrino bi-tri-maximal mixing, Nucl. Phys. B 733 (2006) 31 [hep-ph/0507176] [INSPIRE].

[70] M.L. López-Ibáñez, A. Melis, M.J. Pérez and O. Vives, Slepton non-universality in the flavor-effective MSSM, JHEP 11 (2017) 162 [Erratum ibid. 04 (2018) 015] [arXiv: 1710.02593] [INSPIRE].

[71] I. de Medeiros Varzielas, G.G. Ross and J. Talbert, A unified model of quarks and leptons with a universal texture zero, JHEP 03 (2018) 007 [arXiv:1710.01741] [INSPIRE].

[72] I. De Medeiros Varzielas, M.L. López-Ibáñez, A. Melis and O. Vives, Controlled flavor violation in the MSSM from a unified $\Delta(27)$ flavor symmetry, JHEP 09 (2018) 047 [arXiv: 1807.00860] [INSPIRE].

[73] S. Antusch and M. Spinrath, Quark and lepton masses at the GUT scale including SUSY threshold corrections, Phys. Rev. D 78 (2008) 075020 [arXiv: 0804.0717] [INSPIRE].

[74] S. Antusch and M. Spinrath, New GUT predictions for quark and lepton mass ratios confronted with phenomenology, Phys. Rev. D 79 (2009) 095004 [arXiv:0902.4644] [INSPIRE].

[75] S. Antusch, L. Calibbi, V. Maurer, M. Monaco and M. Spinrath, Naturalness and GUT scale Yukawa coupling ratios in the CMSSM, Phys. Rev. D 85 (2012) 035025 [arXiv: 1111.6547] [INSPIRE].

[76] S. Antusch, S.F. King and M. Spinrath, GUT predictions for quark-lepton Yukawa coupling ratios with messenger masses from non-singlets, Phys. Rev. D 89 (2014) 055027 [arXiv:1311.0877] [INSPIRE].

[77] ATLAS collaboration, Combined search for the Standard Model Higgs boson using up to $4.9 \mathrm{fb}^{-1}$ of pp collision data at $\sqrt{\mathrm{s}}=7 \mathrm{TeV}$ with the ATLAS detector at the LHC, Phys. Lett. B 710 (2012) 49 [arXiv:1202.1408] [INSPIRE].

[78] CMS collaboration, Combined results of searches for the Standard Model Higgs boson in pp collisions at $\sqrt{s}=7 \mathrm{TeV}$, Phys. Lett. B 710 (2012) 26 [arXiv:1202.1488] [INSPIRE].

[79] H. Baer, V. Barger and A. Mustafayev, Implications of a 125 GeV Higgs scalar for LHC SUSY and neutralino dark matter searches, Phys. Rev. D 85 (2012) 075010 [arXiv:1112.3017] [INSPIRE].

[80] A. Arbey, M. Battaglia, A. Djouadi, F. Mahmoudi and J. Quevillon, Implications of a 125 GeV Higgs for supersymmetric models, Phys. Lett. B 708 (2012) 162 [arXiv:1112.3028] [INSPIRE].

[81] J.-J. Cao, Z.-X. Heng, J.M. Yang, Y.-M. Zhang and J.-Y. Zhu, A SM-like Higgs near $125 \mathrm{GeV}$ in low energy SUSY: a comparative study for MSSM and NMSSM, JHEP 03 (2012) 086 [arXiv:1202.5821] [INSPIRE].

[82] M. Carena, S. Heinemeyer, O. Stål, C.E.M. Wagner and G. Weiglein, MSSM Higgs boson searches at the LHC: benchmark scenarios after the discovery of a Higgs-like particle, Eur. Phys. J. C 73 (2013) 2552 [arXiv:1302.7033] [INSPIRE].

[83] G. Barenboim, C. Bosch, M.L. López-Ibáñez and O. Vives, Eviction of a $125 \mathrm{GeV}$ "heavy"-Higgs from the MSSM, JHEP 11 (2013) 051 [arXiv:1307.5973] [INSPIRE]. 
[84] G. Barenboim, C. Bosch, M.L. López-Ibáñez and O. Vives, Improved $\tau$-weapons for Higgs boson hunting, Phys. Rev. D 90 (2014) 015003 [arXiv:1311.7321] [INSPIRE].

[85] Y. Okada, M. Yamaguchi and T. Yanagida, Upper bound of the lightest Higgs boson mass in the minimal supersymmetric Standard Model, Prog. Theor. Phys. 85 (1991) 1 [INSPIRE].

[86] Y. Okada, M. Yamaguchi and T. Yanagida, Renormalization group analysis on the Higgs mass in the softly broken supersymmetric Standard Model, Phys. Lett. B 262 (1991) 54 [INSPIRE].

[87] J.R. Ellis, G. Ridolfi and F. Zwirner, Radiative corrections to the masses of supersymmetric Higgs bosons, Phys. Lett. B 257 (1991) 83 [InSPIRE].

[88] J.R. Ellis, G. Ridolfi and F. Zwirner, On radiative corrections to supersymmetric Higgs boson masses and their implications for LEP searches, Phys. Lett. B 262 (1991) 477 [INSPIRE].

[89] H.E. Haber and R. Hempfling, Can the mass of the lightest Higgs boson of the minimal supersymmetric model be larger than $m_{Z}$ ?, Phys. Rev. Lett. 66 (1991) 1815 [INSPIRE].

[90] S.P. Martin, A supersymmetry primer, hep-ph/9709356 [INSPIRE].

[91] H. Baer, V. Barger and M. Savoy, Upper bounds on sparticle masses from naturalness or how to disprove weak scale supersymmetry, Phys. Rev. D 93 (2016) 035016 [arXiv: 1509.02929] [INSPIRE].

[92] T. Moroi, The muon anomalous magnetic dipole moment in the minimal supersymmetric Standard Model, Phys. Rev. D 53 (1996) 6565 [Erratum ibid. D 56 (1997) 4424] [hep-ph/9512396] [INSPIRE].

[93] L.J. Hall, V.A. Kostelecky and S. Raby, New flavor violations in supergravity models, Nucl. Phys. B 267 (1986) 415 [InSPIRE].

[94] F. Gabbiani, E. Gabrielli, A. Masiero and L. Silvestrini, A complete analysis of FCNC and CP constraints in general SUSY extensions of the Standard Model, Nucl. Phys. B 477 (1996) 321 [hep-ph/9604387] [INSPIRE].

[95] A.J. Buras, A. Romanino and L. Silvestrini, $K \rightarrow \pi \nu \bar{\nu}:$ a model independent analysis and supersymmetry, Nucl. Phys. B 520 (1998) 3 [hep-ph/9712398] [InSPIRE].

[96] M. Misiak, S. Pokorski and J. Rosiek, Supersymmetry and FCNC effects, Adv. Ser. Direct. High Energy Phys. 15 (1998) 795 [hep-ph/9703442] [INSPIRE].

[97] A. Crivellin, Z. Fabisiewicz, W. Materkowska, U. Nierste, S. Pokorski and J. Rosiek, Lepton flavour violation in the MSSM: exact diagonalization vs. mass expansion, JHEP 06 (2018) 003 [arXiv: 1802.06803] [INSPIRE].

[98] A. Dedes, M. Paraskevas, J. Rosiek, K. Suxho and K. Tamvakis, Mass insertions vs. mass eigenstates calculations in flavour physics, JHEP 06 (2015) 151 [arXiv:1504.00960] [INSPIRE].

[99] W. Porod, SPheno, a program for calculating supersymmetric spectra, SUSY particle decays and SUSY particle production at $e^{+} e^{-}$colliders, Comput. Phys. Commun. 153 (2003) 275 [hep-ph/0301101] [INSPIRE].

[100] W. Porod and F. Staub, SPheno 3.1: extensions including flavour, CP-phases and models beyond the MSSM, Comput. Phys. Commun. 183 (2012) 2458 [arXiv:1104.1573] [INSPIRE]. 
[101] H. Baer, A. Belyaev, T. Krupovnickas and A. Mustafayev, SUSY normal scalar mass hierarchy reconciles $(g-2)_{\mu}, b \rightarrow s \gamma$ and relic density, JHEP 06 (2004) 044 [hep-ph/0403214] [INSPIRE].

[102] MEG collaboration, Search for the lepton flavour violating decay $\mu^{+} \rightarrow e^{+} \gamma$ with the full dataset of the MEG experiment, Eur. Phys. J. C 76 (2016) 434 [arXiv:1605.05081] [INSPIRE].

[103] MEG II collaboration, The design of the MEG II experiment, Eur. Phys. J. C 78 (2018) 380 [arXiv: 1801.04688 ] [INSPIRE].

[104] SINDRUM collaboration, Search for the decay $\mu^{+} \rightarrow e^{+} e^{+} e^{-}$, Nucl. Phys. B 299 (1988) 1 [INSPIRE].

[105] A. Blondel et al., Research proposal for an experiment to search for the decay $\mu \rightarrow$ eee, arXiv: 1301.6113 [INSPIRE].

[106] Mu2E collaboration, Mu2e technical design report, arXiv:1501.05241 [INSPIRE].

[107] BABAR collaboration, Searches for lepton flavor violation in the decays $\tau^{ \pm} \rightarrow e^{ \pm} \gamma$ and $\tau^{ \pm} \rightarrow \mu^{ \pm} \gamma$, Phys. Rev. Lett. 104 (2010) 021802 [arXiv:0908.2381] [INSPIRE].

[108] T. Aushev et al., Physics at super B factory, arXiv:1002.5012 [InSPIRE].

[109] BELLE collaboration, Search for lepton-flavor-violating tau decays into a lepton and a vector meson, Phys. Lett. B 699 (2011) 251 [arXiv:1101.0755] [INSPIRE].

[110] M. Graesser and S.D. Thomas, Supersymmetric relations among electromagnetic dipole operators, Phys. Rev. D 65 (2002) 075012 [hep-ph/0104254] [INSPIRE].

[111] Z. Chacko and G.D. Kribs, Constraints on lepton flavor violation in the MSSM from the muon anomalous magnetic moment measurement, Phys. Rev. D 64 (2001) 075015 [hep-ph/0104317] [INSPIRE].

[112] X.-J. Bi, Y.-P. Kuang and Y.-H. An, Muon anomalous magnetic moment and lepton flavor violation in MSSM, Eur. Phys. J. C 30 (2003) 409 [hep-ph/0211142] [INSPIRE].

[113] G. Isidori, F. Mescia, P. Paradisi and D. Temes, Flavour physics at large $\tan \beta$ with a bino-like LSP, Phys. Rev. D 75 (2007) 115019 [hep-ph/0703035] [INSPIRE].

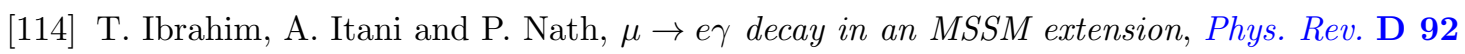
(2015) 015003 [arXiv: 1503.01078] [inSPIRE].

[115] J. Kersten, J.-H. Park, D. Stöckinger and L. Velasco-Sevilla, Understanding the correlation between $(g-2)_{\mu}$ and $\mu \rightarrow e \gamma$ in the MSSM, JHEP 08 (2014) 118 [arXiv:1405.2972] [INSPIRE].

[116] F. Staub, SARAH, arXiv:0806.0538 [INSPIRE].

[117] F. Staub, T. Ohl, W. Porod and C. Speckner, A tool box for implementing supersymmetric models, Comput. Phys. Commun. 183 (2012) 2165 [arXiv:1109.5147] [INSPIRE].

[118] F. Staub, SARAH 4: a tool for (not only SUSY) model builders, Comput. Phys. Commun. 185 (2014) 1773 [arXiv: 1309.7223] [INSPIRE].

[119] S. Antusch, J. Kersten, M. Lindner, M. Ratz and M.A. Schmidt, Running neutrino mass parameters in see-saw scenarios, JHEP 03 (2005) 024 [hep-ph/0501272] [INSPIRE]. 
[120] ATLAS collaboration, Searches for electroweak production of supersymmetric particles with compressed mass spectra in $\sqrt{s}=13 \mathrm{TeV}$ pp collisions with the ATLAS detector, ATLAS-CONF-2019-014, CERN, Geneva, Switzerland (2019).

[121] WMAP collaboration, Seven-year Wilkinson Microwave Anisotropy Probe (WMAP) observations: sky maps, systematic errors and basic results, Astrophys. J. Suppl. 192 (2011) 14 [arXiv: 1001.4744] [INSPIRE].

[122] H. Baer, V. Barger, P. Huang and X. Tata, Natural supersymmetry: LHC, dark matter and ILC searches, JHEP 05 (2012) 109 [arXiv:1203.5539] [INSPIRE].

[123] K.J. Bae, H. Baer, V. Barger, M.R. Savoy and H. Serce, Supersymmetry with radiatively-driven naturalness: implications for WIMP and axion searches, Symmetry 7 (2015) 788 [arXiv: 1503.04137] [INSPIRE].

[124] Muon G-2 collaboration, Muon $(g-2)$ technical design report, arXiv:1501.06858 [INSPIRE].

[125] M. Abe et al., A new approach for measuring the muon anomalous magnetic moment and electric dipole moment, PTEP 2019 (2019) 053C02 [arXiv: 1901.03047] [INSPIRE]. 